\title{
VERTIGES ET PROMESSES DE LA COMPLEXITÉ... QUELLES MÉTAMORPHOSES POUR NOS DÉMOCRATIES DÉSENCHANTÉES ?
}

\author{
Author(s) / Auteur(s) : \\ Janine RENIER \\ Organis'actions \& Systèmes, Consultante en Approche Systémique des Organisations \\ Thérapeute de famille \& de couples, Assistante Sociale, Formatrice à l'IEFC (Institut Européen de Formation \\ Continue), au CFTF (Centre de Formation à la Thérapie de Famille) \& au sein de réseaux psycho-médico- \\ sociaux \\ Membre du groupe méthodologique SPIRAL - Recherche et Anticipation pour la Cohésion Sociale (Conseil de \\ l'Europe - Strasbourg ) devenu réseau Wikispiral/Togheter (territoires de co-responsabilité) \\ renier.janine@gmail.com
}

\begin{abstract}
Résumé :
"La Complexité... désigne tout ce qui échappe, complétement ou partiellement, à notre compréhension, à notre maîtrise" et certaines de ses manifestations contemporaines peuvent se révéler éminemment anxiogènes...

Incertitudes, indéterminations, enchevêtrements, instabilités ; menaces écologiques, crise de la civilisation... hyper-modernité,

Ruptures meurtrières, inter-rétroactions, aléas; crashs bancaires, attentats, violences, dictatures,

Bifurcations destructrices, turbulences, fluctuations dans une crise financière \& économique assortie d'austérité \& d'inégalités accrues.

Nous aborderons l'invention de la démocratie, sous la forme de différentes métaphores: la machine, l'organisme, l'instrument de domination, le cerveau, la culture, le pouvoir.

Peut-on gouverner dans la complexité ? Mais plus encore, dans ces contextes, la démocratie est-elle un leurre, un spectacle pendant que l'essentiel se déroule dans les coulisses? Où le pouvoir est confisqué sous l'emprise de lobbyings et de corruptions? Où la connaissance est réquisitionnée par une caste d'experts parfois eux-aussi inféodés à la pensée néo-libérale et disséminés dans les divisions de vastes bureaucraties mécanistes

La démocratie représentative dispose t'elle en termes organisationnels de la variété requise pertinente pour rencontrer cette imprévisibilité exponentielle, apparente en certains endroits, occultée en d'autres?

Un changement de paradigme est-il exigé dans la manière de faire de la politique ? Changement qui ne pourrait prendre ses racines que dans une nouvelle épistémologie de la complexité en lien avec la démocratie cognitive qu' Edgar Morin appelle de ses væux...où les débats seraient aussi portés par les citoyens : Auto-écoorganisation, récursivité organisationnelle, dialogique, paradoxalité du vivant, consensus/conflictualité, rationalité limitée/multi-rationalité /rético-rationalité, écologie de l'action!

Cette co-construction ne pourrait s'opérer sans une refondation mythique avec l'émergence du sens (à l'échelle planétaire) dans le cadre d'une véritable éthique de la discussion et de reliance.

Dans un environnement complexe et dynamique, les théories de la contingence appellent aussi une démocratie délibérative ascendante, conjuguée à la démocratie représentative (multi niveaux, du local au mondial).

Pour illustrer cette capacité de démocratie continue ou processuelle, les projets d'agir démocratique modélisés par le Conseil de l'Europe (Plans de Cohésion Sociale - Europe - Wallonie) seront convoqués.

Le défi de ces méta-morphoses est donc inséparablement éthique, épistémologique et organis'actionnel !
\end{abstract}

\section{Keywords / Mots-clés :}

Complexité, métaphores, bureaucraties mécanistes, démocratie représentative, variété requise, adhocraties, auto-éco-organisation, éthique de reliance, refondation mythique, théorie de la contingence, démocratie continue, agir démocratique, organis'actions, Plan de Cohésion Sociale. 


\section{Vertiges et promesses de la complexité ${ }^{\prime}$ quelles méta-morphoses pour nos démocraties désenchantées?}

Le radeau de la méduse...œuvre romantique de Géricault, nous accompagne lors de ces journées d'études sur la « démocratie». Ce tableau est réalisé en 1818, peu de temps après la restauration de Louis XVIII sur le trône de France.

Le ROI n'a t'il pas désigné comme responsable du pilotage d'un bateau, par pur népotisme, un capitaine incompétent qui mène à la mort des centaines de personnes?

Symbole de la dérive du peuple français gouverné par un ROI réactionnaire, auréolé de son pouvoir absolu de droit divin, pour les uns? Interrogation sur le sens de la vie au travers la résistance farouche des hommes face aux forces de la nature, pour les autres?

Sommes nous vraiment « sur le radeau de la Méduse » ou comment trouver ensemble, lors de ces journées, des balises éthiques, épistémologiques et organis'actionnelles ....... vers un possible ré-enchantement des démocraties?

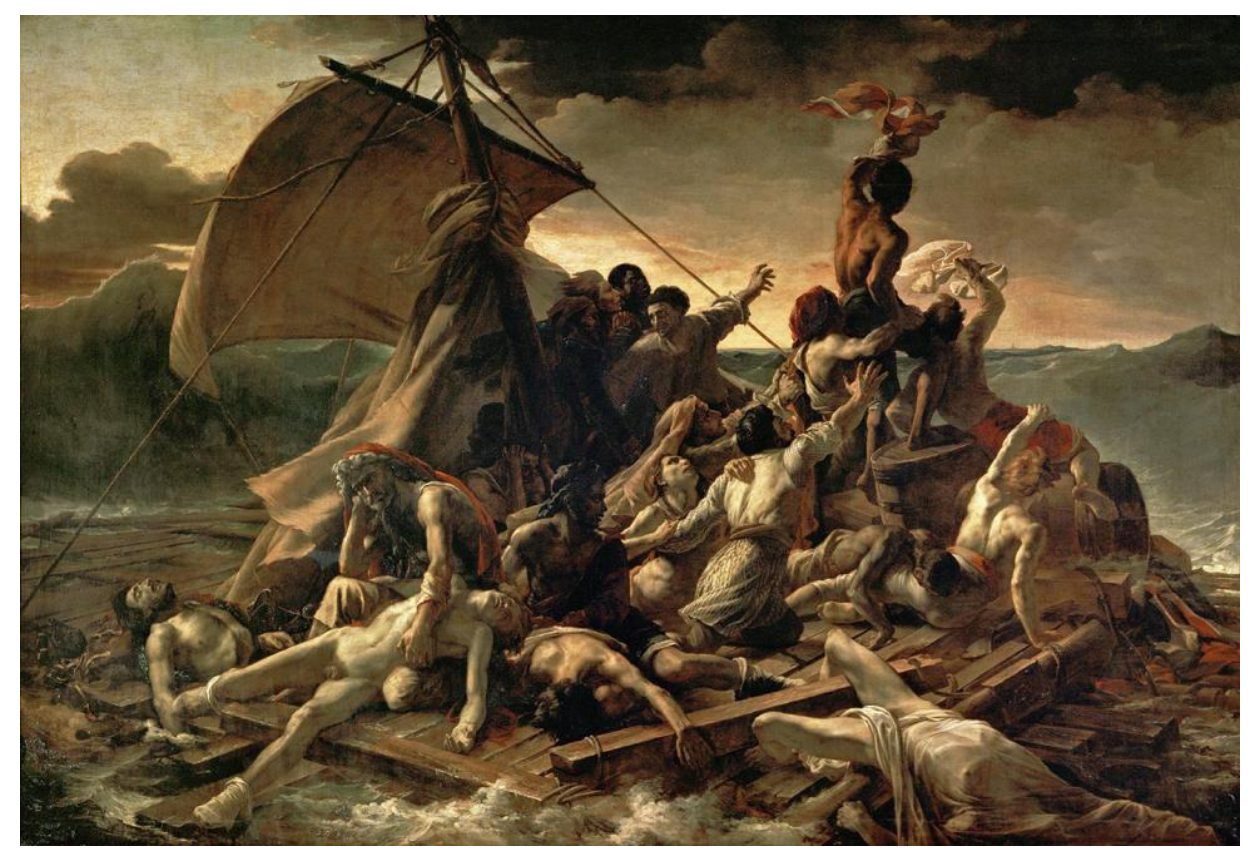

1 BENKIRANE Réda : la complexité, vertiges et promesses, 18 histoires de sciences, le Pommier, 2002. 


\section{PLAN}

1) Vertiges tourbillonnaires dans la complexité.

2) Démocratie, entre Amour et Désamour

3) Les métaphores ... et l'invention de la démocratie.

- 3.1. La machine - paradigme analytique - bureaucraties mécanistes - rationalité limitée - pouvoir classique

- 3.2. L'organisme - paradigme systémique - variété des espèces d'organisations, ouvertes sur l'environnement - principe de la variété requise - multi rationalité/rético-rationalité - théorie de la contingence - pouvoir émergeant

- 3.3. L'instrument de domination : Néolibéralisme: marché total et «société de marché » - darwinisme social - technocraties - exemple de la marchandisation du travail social - soft tyrannie

- 3.4. Le cerveau - vers le paradigme de la complexité - hologramme, récursivité organisationnelle, dialogique, auto-éco-organisation - pilotage multidirectionnel

- 3.5. La culture - le registre mythique - co-construction par le sens intelligence collective - organisation apprenante - Ethique de reliance

- 3.6. Le pouvoir - cette métaphore croisant de manière transversale les 5 premières.

4) Renouveau démocratique : comment penser - agir la démocratie et la citoyenneté dans la complexité? Diverses voies: réformes institutionnelles, résistances, vers des démocraties délibératives/ cognitives - continues.

5) Plans de cohésion sociale en Wallonie et en Europe, les territoires de coresponsabilités ... démocraties délibératives -

6) Quel devenir ? Quelle démocratie? DEMAIN 


\section{1) Vertiges tourbillonnaires dans la complexité}

Si nous scrutons encore notre environnement, que constatons nous aujourd'hui ? Selon Edgar Morin, une profonde crise de civilisation multi-niveaux sévit partout dans le Monde ... Certaines manifestations contemporaines de cette complexité peuvent se révéler éminemment anxiogènes ... nous donner le tournis et parfois provoquer de réels mouvements de panique, sommes nous sur le radeau de la Méduse?

- Incertitudes, indéterminations, enchevêtrements, instabilités: crise environnementale, menaces écologiques,

- Crise de la civilisation, crise des valeurs ... hyper-modernité, instantanéité des médias,

- Guerres multiples, barbaries, terrorismes, attentats, violences génocides, épidémies, migrations,

- Bifurcations destructrices, turbulences, mondialisation débridée, fluctuations dans un capitalisme financier \& économique mondialisé assorties d'austérité \& d'inégalités accrues, confiscation du bien commun, Hommes politiques désavoués et délégitimés,

- Ruptures meurtrières, aléas, crashs bancaires, corruptions, dettes, dictatures..

Ces enjeux trouvent leur issue dans l'acceptation du principe de "complexité, aujourd'hui encore méconnu, même si beaucoup utilisé.

"La Complexité ... désigne tout ce qui échappe, complétement ou partiellement, à notre compréhension, à notre maîtrise. "C'est bien là le premier niveau de réalité auquel nous convie Dominique GENELOT ${ }^{2}$ qui opère une remarquable lecture multi-niveaux de la complexité sur base des travaux d' Edgar MORIN et de Jean-Louis LE MOIGNE principalement.

Ces nombreux facteurs, propres à notre époque accélèrent encore ce mouvement de complexification. Pourtant, il serait illusoire de vouloir à nouveau tout simplifier pour retourner à un état antérieur.

Dans ces contextes troublés, la démocratie est-elle un leurre, un spectacle pendant que l'essentiel se déroule dans les coulisses ${ }^{3}$ ? Le pouvoir y est confisqué aux politiques élus par les professionnels de la finance et de l'économie (y fleurissent d'inévitables corruptions) et l'emprise des lobbyings y est grandissante. Dans les institutions bureaucratiques \& technocratiques accolées aux gouvernements, la connaissance est aussi réquisitionnée par une caste d'experts ${ }^{4}$ parfois eux-aussi inféodés à la pensée néo-libérale. Ils sont disséminés dans les divisions de vastes bureaucraties mécanistes 5 .

\footnotetext{
2 GENELOT Dominique: Manager dans le complexité, réflexion à l'égard des dirigeants, INSEP CONSULTING Editions, 4eme édition, Paris 2011.

${ }^{3}$ SINTOMER Yves: "La démocratie devient spectacle, pendant que l'essentiel se déroule en coulisses » interview réalisé par ABESCAT Michel, article de Télérama publié le 01/07/2016.

4 ISMARD Paulin: La démocratie contre les experts, I'Univers UH historique, SEUIL, mars 2015.

5 MINTZBERG Henry: Le management : voyage au centre des organisations, éditions d'Organisation, 1989. 
La perméabilité entre secteur privé, hautes administrations, monde politique, médias, élites en tous genres est chaque jour invisible/perceptible.

Vers où allons nous?

Une pensée complexe peut nous permettre de modéliser les gouvernements démocratiques, mais peut-elle aussi nous aiderà co-construire une Société Démocratique revisitée, porteuse d'un avenir meilleur?

\section{2) DEMOCRATIE... entre Amour et Désamour}

Le pouvoir politique, dans nos sociétés modernes, est lié à l'État, même si le politique préexiste en réalité à l'État. La démocratie libérale 6 , parfois appelée démocratie occidentale, est une idéologie politique et une forme de gouvernement dans laquelle la démocratie représentative fonctionne selon les principes du libéralisme, à savoir la protection des droits des minorités et, en particulier, l'individu. Elle est caractérisée par des élections justes, libres et concurrentielles entre plusieurs partis politiques distincts, une séparation des pouvoirs exécutif, législatif et judiciaire, la primauté du droit dans la vie quotidienne dans le cadre d'une société ouverte, et la protection égale des droits de l'homme, des droits et libertés civils, et des libertés politiques pour tous les hommes.

En pratique, les démocraties libérales sont souvent basées sur une constitution, afin de définir les pouvoirs du gouvernement et de consacrer le contrat social.

Après une période d'expansion soutenue tout au long du XXème siècle, la démocratie libérale est devenu le système politique de la majorité des pays développés et une sorte d'idéal... incontournable.

Une démocratie libérale peut prendre diverses formes constitutionnelles. Elle peut reposer sur une république, une monarchie constitutionnelle, un régime présidentiel, semi-présidentiel ou parlementaire.

Abraham LINCOLN définit (1863) la démocratie par ces mots: le gouvernement du peuple, par le Peuple, pour le peuple.

Pourtant nulle part, il n'existe de démocratie directe...aujourd'hui.

Loï BLONDIAUX ${ }^{7}$ analyse avec pertinence l'impérieuse nécessité d'une méta-morphose de la démocratie dans une conférence donnée en 2011: ceux qui l'ont inventé à la fin du $18^{e}$ siècle aux Etats unis et en France ne voulaient pas de la démocratie directe.

\footnotetext{
6 WIKIPEDIA, Démocratie libérale, https://fr.wikipedia.org/wiki/Démocratie libérale

${ }^{7}$ BLONDIAUX Loïc, «Métamorphoses de la démocratie: vers une démocratie réelle» Session 2011 des Semaines sociales, "La démocratie une idée neuve». Professeur des Universités au département de science politique de la Sorbonne (Paris I), chercheur au Centre européen de sociologie et de science politique de la Sorbonne (CESSP) et au Centre de recherches politiques de la Sorbonne (CRPS).
} 
Termes forts, mais c'est pourquoi, elle se mit en place sous la forme de la démocratie représentative (défensive), que de nombreux observateurs qualifient aujourd'hui d'oligarchie, avec la prise de conscience que la souveraineté était confisquée par une classe restreinte de privilégiés, une aristocratie élective.

Nous sommes très loin de cette Athènes (VIème siècle avant Jésus Christ) où la démocratie directe fleurissait grâce au tirage au sort et dont de nombreux chercheurs, philosophes politiques, sociologues, citoyens ... nous chantent aujourd'hui encore les louanges. Mythique Athènes, pourrions nous dire?

Ainsi donc, notre résonance émotionnelle envers la démocratie oscille entre amour et désamour: le pire système de gouvernement, à l'exception de tous les autres qui ont pu être expérimentés dans l'histoire, disait Winston Churchill.

Ce mythe fondateur de la DEMOCRA TIE reste aujourd'hui vivace de façon paradoxale: tout le monde s'y réfère encore mais elle est en même temps affublée de noms de plus en plus disqualifiant : fausse démocratie-CASTORIADIS Cornélius, démocratie myope Pierre ROSANVALLON, démocratie fatiguée - David VAN REYBROUCK, démocratie désenchantée - Marcel GAUCHET, post démocratie - Colin CROUCH, dé-démocratie Wendy Brown, conduisant parfois au malaise dans la démocratie -Jean-Pierre LE GOFF et jusqu'à la haine de la démocratie - Jacques RANCIERE, ou encore à la médiocratie Alain DENEAULT ! La démocratie, nouvel habit de la tyrannie, nous disent même Natacha POLONY \& le comité ORWELL...

Et cette liste est loin d'être exhaustive!

Peut-on gouverner dans la complexité? La démocratie représentative peut-elle se révéler la configuration organisationnelle pertinente pour rencontrer celle dernière?

La société contemporaine vit donc de grands bouleversements, ils mettent en évidence le fait que les réponses données jusqu'à aujourd'hui ne font désormais plus sens dans cet environnement complexe, évolutif où les changements sont constants et de plus en plus rapides.

Allons donc vers une compréhension de la naissance de la démocratie et vers son évolution au fil du temps.

\footnotetext{
${ }^{8}$ POLONY Natacha \& le comité ORWELL, Bienvenue dans le pire des mondes, le triomphe de la soft tyrannie, PLON, $\underline{2016}$ 


\section{3) Métaphores ....invention de la démocratie et des organisations qui l'accompagnent}

Le réel existe, mais nous l'approchons au travers nos représentations et nos modélisations. Le « réel » dont nous parlons n'est pas le réel, mais seulement notre point de vue sur le réel. Il s'agit donc bien d'un réel construit.

Jean-Louis Le MOIGNE a résumé ce mécanisme du système de représentation en 3 points repris par Dominique GENELOT':

$\rightarrow$ Contexte

$\rightarrow$ Grille d'interprétation du monde

$\rightarrow$ Quelles Intentions, visions du futur du/des modélisateur(s) ???

Gareth MORGAN ${ }^{10}$, de manière poétique, va lui illustrer notre façon de penser, de voir, d'agir, au sein des organisations, à l'aide de métaphores qui produisent toujours une sorte de connaissance analogique \& paradoxale du vivant, toutes teintées d'une grande force de créativité et d'intuition. Je vais lui en emprunter certaines d'entre elles et hybrider ces deux lectures.

\section{3) 1 - MACHINE}

\section{Contexte socio-politique, économique, éthique}

La démocratie commence à triompher à la fin du XXVIIIème Siècle $e^{11}$

La révolte des colonies anglaises d'Amérique du Nord donne naissance à la Déclaration des droits de 1776, qui sert de base à la Constitution démocratique des États-Unis.

En Europe, le deficit bugétaire des Etats est chronique pour faire face à des Guerres couteuses, le peuples sont un peu partout opprimés par les classes dirigeantes (Roi , Aristocraties, Clergé).

La Révolution française de 1789 ébranle tout l'édifice des monarchies de droit divin (importance donc de la religion) où le pouvoir est absolu, linéaire, descendant, autocratique.

Ce pouvoir est de plus en plus remis en question dans la gestion de l'état et dés lors les sociétés d'ordre et de privilèges de l'Ancien Régime sont bousculées.

Lors de cette révolution se forge la Déclaration Universelle des Droits de l'homme et du citoyen de portée plus universelle.

La liberté est définie comme une faculté inhérente à la personne humaine et se réalise pleinement à travers la reconnaissance de droits naturels, inaliénables et sacrés. Il s'agit bien de nouvelles valeurs morales.

\footnotetext{
9 GENELOT Dominique: Manager dans le complexité, réflexion à l'égard des dirigeants, INSEP CONSULTING Editions, 4eme édition, Paris 2011, pp. 95 à 114

${ }^{10}$ MORGAN Gareth: IMAGES de l'organisation, les presses de l'université LAVAL, éditions ESKA, 1989.

${ }^{11}$ LAROUSSE, démocratie http://www.larousse.fr/encyclopedie/divers/démocratie/41420
} 


\section{Grille d'interprétation du monde}

\section{Paradigme analytique}

La grande façon d'étudier le monde à cette époque s' apparente à la démarche cognitive analytique classique, présumée objective (Héritage de Descartes en France), dans laquelle l'objet à connaitre est divisé en différentes parties. L'avantage de cette pensée est la connaissance des détails, la prévisibilité, l'exhaustivité.

\section{Métaphore de la machine}

Pour illustrer comment ceci s'est traduit en termes « organisationnels», MORGAN va utiliser la métaphore de la machine: système fermé, clos et disctinct de l'environnement, fait de parties élémentaires, que l'on décompose en éléments simples, en règles simples de combinaison, avec des causalités linéaires exclusivement.

L'organisation Machine est fiable, précise rationnelle (one best way) elle préconise une vision reductrice de l'homme.

\section{Bureaucratie mécaniste}

Dans le monde de l'entreprise cette organisation Machine va être programmée sur base de l'organisation scientifique du travail (OST), dont l'illustre représentant est Taylor. A côté de l'Etat-Nation et de son gouvernement représentatif, une cohorte de bureaucraties mécanistes remplit une fonction indispensable. Le gouvernement y est donc secondé/ concurrencé... par une administration centrale avec un élargissement des Services publics. L'appareil bureaucratique est une sorte "d'ogre gigantesque » sous la forme d'une vaste organisation divisionnalisée à l'échelle de la Nation.

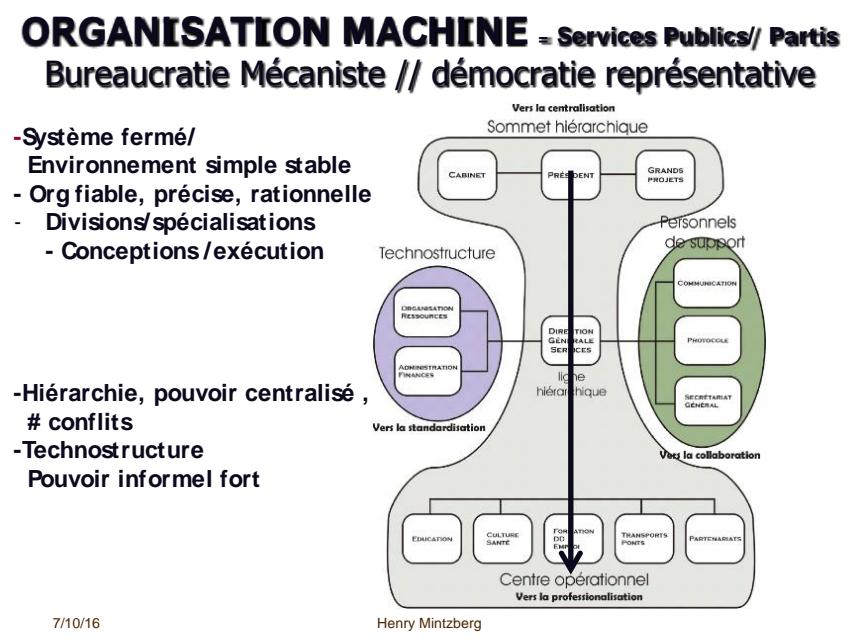

Les structures sont lourdes, couteuses, réparties en silos, compartimentées en services différents qui s'ignorent la plupart du temps, escortées de hiérarchies, de grades, de statuts ..... et qui ignorent leurs clients/usagers/citoyens avec qui elles communiquent peu. Une sorte d'immobilisme funeste 
$\rightarrow$ Pouvoir classique dans la bureaucratie mécaniste.

La vision mécaniste requiert une structure hiérarchisée, une centralisation des décisions, une decomposition artificielle de la conception et de l'exécution. Les décisions sont prises au sommet et exécutées à la base; une direction autoritaire, une discipline des fonctionnaires, la quasi sacralisation d'un pouvoir descendant. L'AUTORITE y est encore admise et même revendiquée. Faut-il dire que cela correspond au modèle de l'autorité patriarcale de l'époque?

Le contrôle y est omniprésent et le pouvoir quasiment exclusivement descendant, mais aussi de type aussi rationnel-légal qui se fonde aussi beaucoup sur l'écriture et les procédures: « nous sommes censés faire comme cela».

Les conflits existent mais ont tendance a y être contrôlés et/ou niés puisque l'obéissance y est la règle. ${ }^{12}$

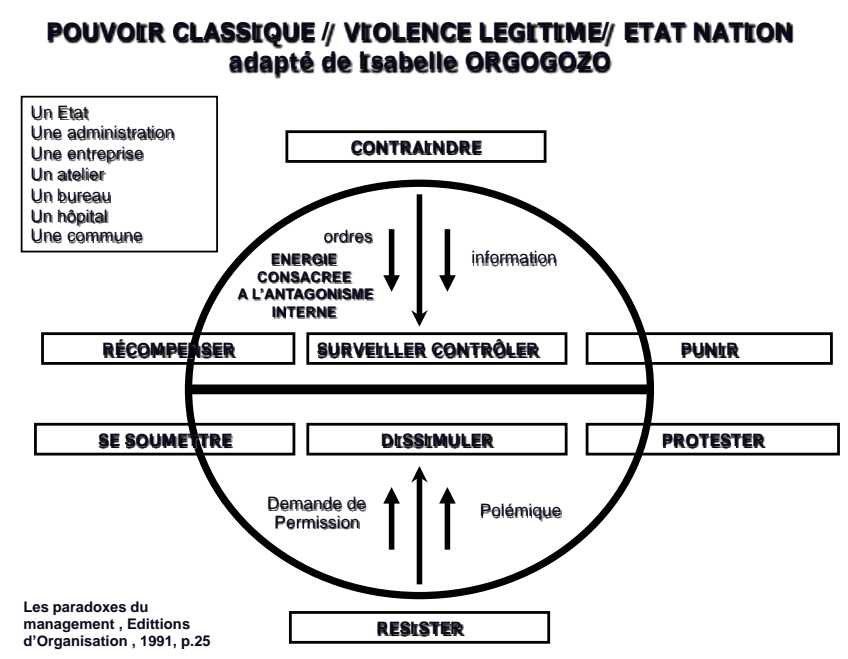

\section{$\rightarrow$ Rationalité limitée}

Qui plus est, dans ce type de configuration organisationnelle, la rationalité y est limitée, selon l'expression de Herbert SIMON ${ }^{13} \& 14$, prix Nobel d'économie. Explorant le parallèle existant entre la prise de decision individuelle et la prise de decision organisationelle, il estime que cette dernière ne peut jamais être entièrement rationnelle, parce que les membres de l'organisation ne disposent que d'un savoir et d'une information limités et des compétences elles aussi parcellaires. Les différents secteurs fonctionnent souvent à partir d'images différentes de la situation globale, sur base de leur systèmes de représentation

L'intelligence collective est loin d'être à l'œuvre dans ces structures en silos et en paliers hiérarchiques divers.

\footnotetext{
12 ORGOGOZO Isabelle: les paradoxes du management, P.25, Les EDITIONS D'ORGANISATION, 1991

${ }^{13}$ SIMON Herbert Alexander: Les sciences de l'artificiel, collection Folio essais ( $\left.n^{\circ} 435\right)$ Gallimard, 15/01/2004.

${ }^{14}$ SIMON Herbert Alexander: Sur la Complexité des systèmes complexes, dans « les Introuvables en langue française de SIMON Herbert Alexander. », document $n^{\circ} 6$, Université Carnegie-Mellon, Pittsburgh
} 


\section{Quelles intentions et des visions du futur du/des modélisateur(s) ???}

\section{$\rightarrow$ Démocratie représentative}

La démocratie directe est l'une des formes premières de la démocratie dans laquelle le peuple exerce directement le pouvoir politique, alors que dans une démocratie représentative, il l'exerce de manière indirecte. Cette dernière est fondée sur le principe selon lequel seul un corps de représentants élus peut incarner véritablement la volonté nationale. Ces deux visions s'opposeront suivant les époques.

Comme évoqué plus haut, dans I'Antiquité et en particulier au VIe siècle avant notre ère (ATHENES), des cités ou des groupes sociaux étaient organisés en démocratie directe. Jean-Jacques ROUSSEAU (1712-1778) estime que la démocratie ne peut être que directe en se fondant sur les droits naturels des êtres humains et sur le pacte social qui les unit. Cependant, au XVIIème et au XVIIIème siècles, de nombreux penseurs considèrent que la démocratie directe n'est applicable que dans de petits pays, avec peu d'habitants et ayant une structure sociale homogène. De nombreux hommes politiques de l'époque aux Etats-Unis et en France ne voulaient pas de la démocratie directe. BLONDIAUX, évoquant SIEYES évoque les freins: elle leur faisait peur ; ... elle n'est pas apte à fonctionner pour de grands états, ....les citoyens ne sont pas des professionnels de la politique et ....surtout ils n'ont pas les compétences requises pour gouverner (dixit dejà Montesquieu). Ils s'écarteront de cette conception, impossible à mettre en ceuvre, selon eux, dans les grands États et dans les sociétés modernes complexes. Ils optent donc pour la démocratie représentative.

\section{Pouvoir classique paradoxal, ascendant/descendant dans la démocratie}

De supposé-ascendant par les élections, le pouvoir se révèle en réalité unilatéral et surtout descendant, seul un petit nombre gouverne, puisque l'élection consacre une oligarchie ou aristocratie élective (Bernard MANIN) ${ }^{15}$. Celle-là même qui est maintenant dénoncée un peu partout.

\section{$\rightarrow$ Démocratie représentative : un hybride}

Pourtant si ce pouvoir reste exclusivement descendant, dans les bureaucraties mécanistes ( qui devraient être leur bras armé), au niveau sociétal dans l'incarnation de la « démocratie » élective et dans le gouvernement il s'agit d'un mixte ou d'un hybride, puisque ce pouvoir provisoire est accordé par les citoyens ... qui ne gouvernent pas dans ce régime, d'autres gouvernent pour eux, qu'ils contrôlent et sanctionnent par intermittences ... par les élections. Les élites qui gouvernent pour eux ne sont pas strictement tenus de réaliser les voeux. C'est pourquoi, ce type de gouvernement combine des éléments démocratiques et des éléments non démocratiques.

\footnotetext{
${ }^{15}$ LANDEMORE Hélène, "la démocratie représentative est elle réellement démocratique? debat avec MANIN Bernard \& URBINATI Nadia, New-York en avril 2007, article du 7 mars 2008. 


\section{$\rightarrow$ Démocratie représentative : un oxymore}

Mais pour l'époque, la grille d'interprétation, le contexte et la vision du futur des modélisateurs est pourtant une avancée par rapport à la phase précédente où le pouvoir était concentré dans les mains d'un Roi de droit divin et omnipotent.

Claude Lefort ${ }^{16}$ de nous dire : « la démocratie pour moi ne peut se reduire à un système juridico-politique, parce que beaucoup plus profondemment, elle procède pour la première fois d'un consentement tacite à la pluralité des intêrets, des opinions, des croyances et même au consentement du conflit. C'est même la reconnaissance du conflit, le refus d'une autorité inconditionnée, le refus du pouvoir incarné dans un monarque, qui est à l'origine du mouvement démocratique. »

La démocratie représentative est donc dejà un oxymore. Les citoyens incompétents sont assez avisés pour désigner les bons représentants. Mais en quelque sorte, ils reçoivent les miettes d'un pseudo-pouvoir. Concession intelligente, consciente ou inconsciente à un peuple qui revendique une part de « co-decision».

Mais, dans toute la société, les usagers/citoyens dépendent de divers services publics, de bureaucraties mécanistes, d'administrations divisionnalisées, où le pouvoir des élus tente encore de s'imposer de manière souvent incontestée.

\section{3) 2 - ORGANISME}

\section{Contexte socio-politique, économique, éthique}

La première moitié du XXème s. est marquée par l'instauration du suffrage universel dans la majorité des pays européens

Les valeurs de la démocratie pluraliste et libérale sont toutefois rejetées par deux types de régimes: d'une part, par les dictatures fasciste d'Italie et nazie d'Allemagne (ainsi que par d'autres régimes autoritaires comme alors en Espagne ou au Portugal), et, d'autre part, par les régimes communistes qui se réclament de la démocratie socialiste, dite « populaire » qui échoue lamentablement avec toutes les horreurs que l'on sait.

Ce nouveau modèle social est fondé sur l'espoir d'une mondialisation heureuse où progrès scientifique, croissance économique, démocratie et conception partagée de la justice sociale s'entrecroisent. « L'esprit de Philadelphie » ${ }^{17}$ instaure la dignité humaine comme pierre angulaire de l'édifice juridique international et met l'économie au service des hommes, la subordonnant au principe de la justice sociale.

Au lendemain de la seconde guerre mondiale, sont signés les accords de Bretton Woods (Banque Mondiale et FMI), la consécration internationale des principes démocratiques, en particulier des droits de l'homme, se traduit par la Déclaration universelle des droits

\footnotetext{
${ }^{16}$ LEFORT Claude, " Entretien avec Claude LEFORT " dans Philosophies de notre temps, Editions Sciences humaines , 2000, PP 167 à 173.

${ }^{17}$ Déclaration de Philadelphie concernant les buts et objectifs de l'Organisation internationale du travail - OIT» (on dira « Déclaration», en abréviation) adoptée en mai 1944
} 
de l'homme adoptée le 10 décembre 1948 par l'Assemblée générale des Nations unies. Suivront, en 1966, deux pactes, I'un relatif aux droits civils et politiques, et l'autre aux droits économiques, sociaux et culturels.

Dans le cadre européen, la Convention, européenne des droits de l'homme est signée à Rome le 4 novembre 1950.

Cependant, I'URSS a imposé son système politique et économique à la majeure partie des pays de l'Europe orientale et centrale, instaurant un régime « totalitaire » (fondé sur une base autoritaire et policière, tout mouvement critique étant jugé subversif). Dans le contexte de la guerre froide opposant le bloc de l'Est et le bloc des démocraties occidentales, la « version socialiste de la démocratie » est également appliquée en Chine et dans un certain nombre de pays en développement.

En 1989 : Chute du mur de Berlin, la démocratie de type libérale, apparaît alors comme un horizon indépassable

Les révolutions démocratiques de 1989 dans les pays de l'Europe de l'Est et la dissolution de l'URSS (1991) mettent fin à cet affrontement. La conversion des régimes communistes à l'économie de marché aurait pourtant contribué à diluer le principe de justice sociale?

Dès lors, le pluralisme libéral peut revendiquer l'exclusivité du modèle démocratique. Mais elle apparaît toujours comme un oxymore.

Mais, même si, au plan des valeurs, le modèle de la démocratie pluraliste s'est imposé, plusieurs régimes exercent en fait dans le monde un pouvoir autoritaire, en appliquant le libéralisme économique (Pinochet au Chili ...)

\section{Grille d'interprétation du monde}

\section{$\rightarrow$ Paradigme Systémique}

Comment la «grille d'interprétation incorporée en nous » évolue peu à peu. Les théories sociologiques sur les organisations s'enrichissent de nouvelles découvertes : théories de la motivation, analyse des systèmes sociotechniques (avec l'interdépendance des besoins humains et techniques), gestion des ressources humaines.

Les travaux du biologiste, Ludwig VON BERTALANFFY vont faire changer les représentations: l'objet à analyser n'est plus réduit à ses éléments constitutifs, mais c'est lui même un système complexe ouvert sur son environnement, sous-système d'un ensemble plus vaste, et lui-même constitué de systèmes plus restreints ${ }^{18}$.

Le système observé n'est pas indépendant de l'observateur, il forme système avec lui ; le système est ouvert sur l'environnement, est fait lui-même de systèmes ouverts, où l'on analyse les interactions entre les éléments et avec l'environnement et où apparaît également la causalité circulaire.

L'approche systémique émerge simultanément des deux côtés de l'atlantique dans les années $50 / 60$

\footnotetext{
${ }^{18}$ BONAMI, de HENNIN, BOQUE, LEGRAND, Management des systèmes complexes, De Boeck Université, 1993, Bruxelles, p. 17 


\section{$\rightarrow$ Métaphore de l'organisme}

Appliquée aux organisations, elle les imagine comme des organismes, ouverts comme eux, sur l'environnement. L'accent est mis sur l'environnement dans lequel les organisations existent (environnement immédiat de la tâche ou environnement contextuel plus général), l'organisation est conçue comme ses sous-systèmes reliés entre eux (à la manière des poupées russes) et l'utilisation pragmatique de l'approche systémique conduit aussi à tenter de mettre à jour des similitudes entre différents systèmes.

Apparaissent ensuite le principe de la variété requise, de différenciation et d'intégration et les théories de la contingence.

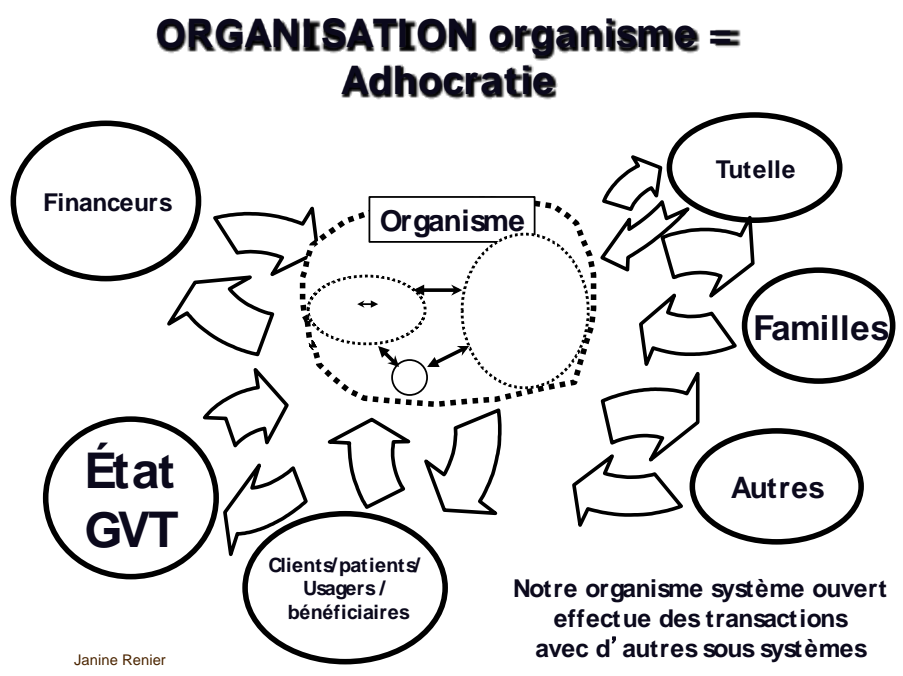

$\rightarrow$ Organisations « organiques » adhocratie, adaptatives, plus souples

Ainsi donc, corrélé à cette nouvelle vision du monde, à un environnement d'avantage en pleine mutation, se complexifiant, bien plus dynamique, nous voyons alors émerger une «variété des espèces d'organisations»; adhocratie, mais structures matricielles, organisations par projet, travail en réseaux, bureaucraties professionnelles tout en souplesse et adaptabilité ....mais les bureaucraties mécanistes n'ont pas disparu pour autant!

Principes de la variété requise (Ashby) 19: multi rationalité/rético rationalité

Plus un système est varié, plus le système qui le pilote doit l'être aussi

La loi de la variété requise est reliée aux concepts de différenciation et dintégration. Cette loi stipule que les mécanismes régulateurs internes d'un système doivent être aussi variés que l'environnement avec lequel il tente de composer, car ce n'est qu'en intégrant la variété requise aux contrôles internes qu'un système peut faire face à la variétés et aux défis de son environnement. Un système qui s'isole de la variété du milieu dans lequel il se trouve tend à s'atrophier, perd de sa complexité et voit

\footnotetext{
${ }^{19}$ MORGAN Gareth: IMAGES de l'organisation, les presses de l'université LAVAL, éditions ESKA, 1989. P 43
} 
disparaître ces traits distinctifs.

C'est ainsi que la variété requise constitue une caractéristique importante de tous les systèmes vivants.

L'environnement social, économique, politique, culturel, écologique... est extrêmement complexe et dynamique, les organisations doivent être capables elles aussi de lire cette variété et pour ce faire, elles doivent intégrer le même niveau de complexité (travail d'équipe \& en réseaux plus horizontaux).

Les bureaucraties mécanistes, conçues sous forme de pyramides, n'arrivent plus à intégrer les changements de l'environnement : la poste, les grands ministères sont des exemples probants. Les services sociaux trop sclérosés et divisionnalisés présentent la même difficulté d'adaptation aux changements externes.

Ce principe de "la variété requise » est corrélée au principe de subsidiarité ${ }^{20}$ qui se traduit par le fait de laisser le contrôle local à ceux qui ont la représentation la plus juste et la plus exhaustive possible de ce qu'il convient de faire, compte tenu de leur perception plus proche et plus pertinente des environnements d'action.

Elle s'appuie sur un principe de confiance (on évite de contrôler a priori ce qu'on peut déléguer à des personnes compétentes et de bonne foi) et un principe de prudence (on $n$ 'intervient pas sur des fonctionnements dont on sait ne pas comprendre toute la complexité).

Deux principes qui se situent au niveau des « programmes officiels» mais qui n'excluent nullement contrôles et évaluations. Le principe de subsidiarité est aussi fréquemment contredit en raison de la prééminence du droit de l'Union européenne, elle même vaste bureaucratie mécaniste \& technocratique.

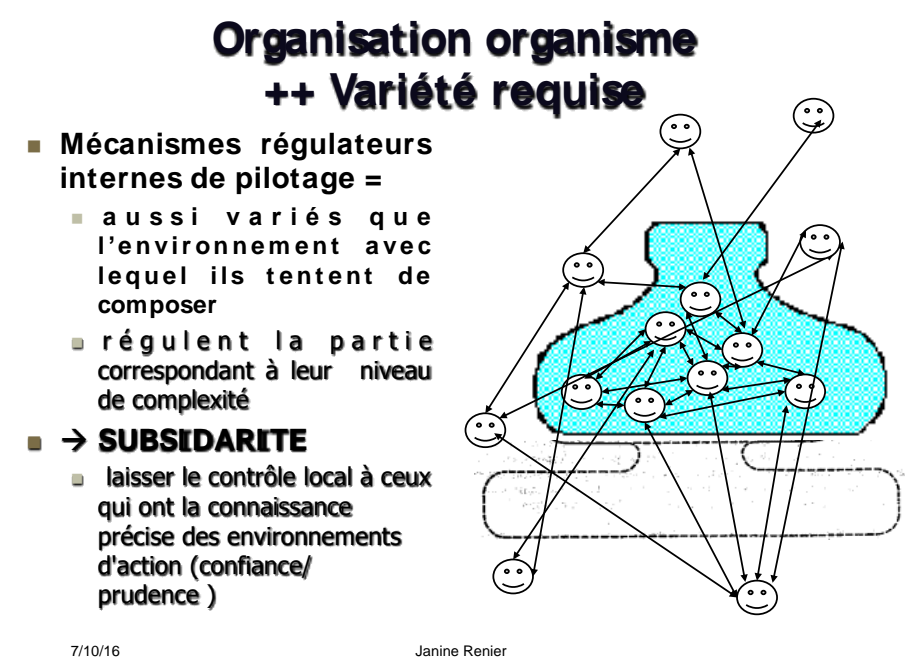

\section{Quelles intentions et des visions du futur du/des modélisateur(s) ???}

Comment au fil du temps, les organisations se reconfigurent-elles autrement sous l'impulsion de leurs modélisateurs? Quelles impérieuses necessités devraient alors

\footnotetext{
${ }^{20}$ Introduction au Management des Universités, http://bricks.univ-lille1.fr/M23/cours/co/chap02_02.html 
s'imposer aux démocraties et aux organisations qui l'accompagnent pour qu'elles puissent devenir « durables » dans un environnement complexe et lui-même évolutif?

\section{$\rightarrow$ Théorie de la contingence : comment adapter l'organisation à l'environnement?}

Une des propositions centrale de l'école de la contingence, est que les facteurs environnementaux rendraient contingentes les structures de l'organisation. Ainsi, contrairement au taylorisme qui s'engage dans la recherche normative de la bonne organisation « en soi », Henry MINTZBERG ${ }^{21}$, le principal représentant de l'école de la contingence, affirme que la structure est liée à la nature de l'environnement, bien qu'elle ne le soit pas de manière mécanique ou déterministe.

La structure dépendant également des buts que se fixent les dirigeants. Dès lors, les mécanismes régulateurs internes d'une organisation doivent être aussi variés que l'environnement avec lequel elle doit composer. Il n'y a donc pas de structure universelle s'adaptant à toutes les situations.

- Plus l'environnement est dynamique, plus la structure est organique.

- Plus l'environnement est complexe plus la structure est décentralisée.

- Plus l'organisation a des terrains diversifiés, plus elle a tendance à se scinder en divisions.

- Une hostilité extrême de l'environnement amène toute organisation à centraliser sa structure de façon temporaire

Hiérarchie bouclée démocratique

Lien avec la complexité de l'environnement et les acteurs

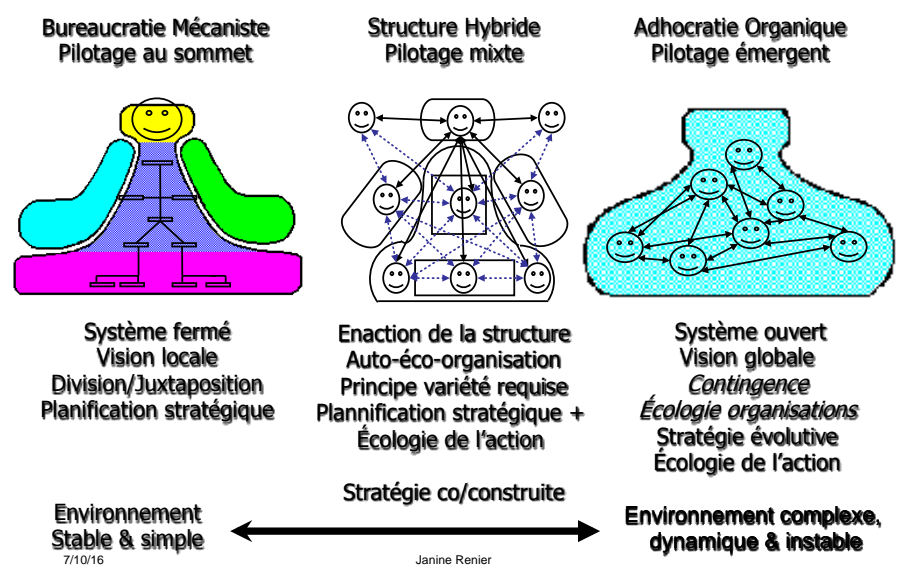

But last and not least, le dernier point de la théorie de la contingence ne va pas cesser de nous interpeller : une hostilité extrême de l'environnement amène toute organisation à centraliser sa structure de façon temporaire, nous pourrions dire en résumé : dans la crise vive le retour du « chef salvateur «???? Nous l'évoquerons encore plus loin

\section{$\rightarrow$ Pouvoir ascendant ou Empowerment}

\footnotetext{
${ }^{21}$ MINTZBERG Henry: Structure et dynamique des organisations, Editions d'Organisation, 1996.
} 
Comment doivent évoluer les organisations pour survivre et comment doivent-elles integrer la direction, le pilotage et le pouvoir dans tous les systèmes. Les organisations vont donc se trouver dans une sorte de double contrainte interne et externe nous dit Claude-Pierre VINCENT ${ }^{22}$, c'est dans ce contexte que les responsables politiques et les managers vont être sommés d'agir, c'est-à-dire d'imprimer au système une direction (contrôle - pilotage -régulation) en tenant compte des acteurs internes du système, des acteurs externes et des environnements.

La direction de tels systèmes ne devrait plus se concevoir exclusivement du haut vers le bas. De nombreux sujets y partagent savoir et pouvoir. La direction est elle- même un réseau, propriété du tout. Ceci se réalise dans un apprentissage collectif où seront réaménagées valeurs, croyances et systèmes de représentation en présence. Et les rationalités deviennent elles aussi plurielles (multi-rationalité ${ }^{23}$ ) ou encore en réseaux (rético-rationalité) 24 .

La conception même du pouvoir au sein de la société, des organisations et de la famille évolue en parallèle, toutes ces structures se doivent d'être «démocratiques», autrement dit, porteuses d'une conception d'un pouvoir partagé. ${ }^{25}$

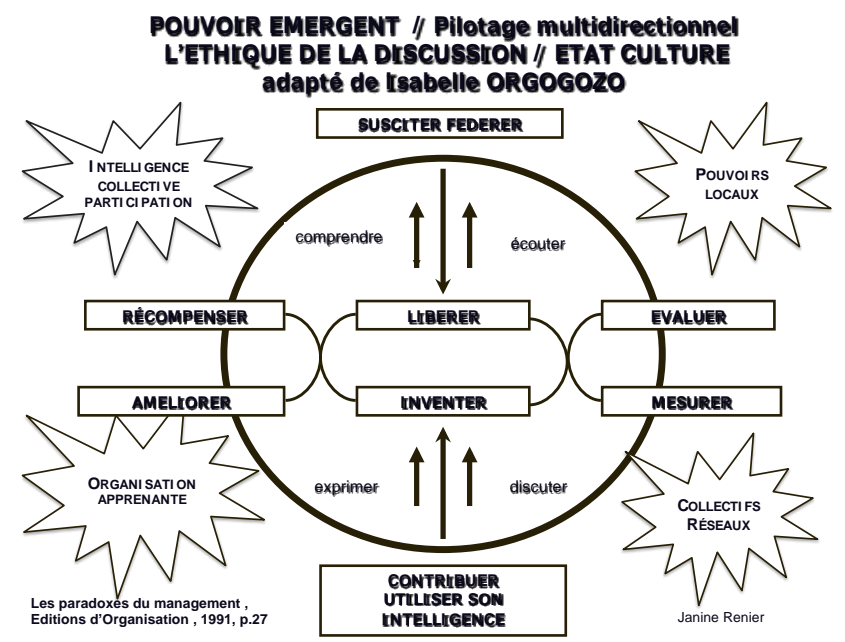

\section{3) 3 - INSTRUMENT DE DOMINATION NEOLIBERALISME}

\section{Contexte socio-politique, économique, éthique}

MYTHE du Néolibéralisme : « marché total » et « société de marché »

"Guerre, crise, guerre: notre monde contemporain s'est forgé durant les cinquante premières années du XXe siècle, au travers d'épreuves qui ont par trois fois laissées

\footnotetext{
22 VINCENT Claude-Pierre. Des systèmes et des Hommes, Editions d'organisation, 1990.

23 GELINAS Arthur: «Evaluation et Multirationalité » Université de Québec à Rimouski, dans PAQUET C. «Pratiques évaluatives » NHP. 1984

24 KERVERN Georges Yves: La culture Réseau éthique et écologie de l'entreprise, Editions ESKA, 1993.

25 ORGOGOZO Isabelle: les paradoxes du management, P.27, Les EDITIONS D'ORGANISA TION, 1991 100
} 
penser que le capitalisme allait s'effondrer: la révolution bolchévique face à l'Europe exsangue de 1918, la grande crise des années trente, la montée du totalitarisme nazi. La force et la modernité du message de KEYNES sont d'avoir élaboré, lors de ces trois échéances tragiques, une réponse réformiste originale, qui plaidait en faveur de l'intervention publique dynamique dans un capitalisme maintenu, mais infléchi dans le sens de la consommation populaire. $»^{26}$

Après le libéralisme, le néo-libéralisme s'est imposé dans les années 1970 en tant que système de pensée ou idéologie et il organise le contexte dans lequel nous vivons quasiment au niveau mondial actuellement.

Les thèses KEYNESIENNES qui préconisaient l'importance du rôle de l'état face au désequilibre du marché, sont abandonnées au profit des thèses de Friedrich HAYEK ${ }^{27}$ ( 1899-1992) et plus tard de Milton FRIEDMAN (1912-2006), chef de file de l'école de Chigaco.

La régle du marché devient absolue, pour HAYEK la selection naturelle prévaut et la justice sociale est illusoire....

Cette idéologie peut se traduire comme « marché total » et/ou « Société de marché ».

Quelques grands penseurs contemporains nous aident à complexifier et à clarifier notre pensée et nous accompagnent dans la construction de notre 'représentation'. Pourquoi cette idéologie se révèle t'elle actuellement toxique?

Certains lui ont reproché d'employer les concepts de la systémique ou des sciences de la complexité comme «l'auto-organisation» d'une manière différente de la leur : ainsi de Jean-Louis Le MOIGNE qui est apparenté au courant épistémologique constructiviste dont Hayek se démarque 28

Pour I'anti-utilitariste Alain CAILLE ${ }^{29}$, la société que prône HAYEK s'apparente à une utopie du «marché » qui, inapplicable dans la réalité, permet de justifier les injustices sociales: «à défaut de l'achat d'une paix sociale par l'État-Providence, l'ordre de marché aurait été balayé depuis longtemps ».

Karl POLANYI (1886-1964) ${ }^{30}$ nous dit que dans presque toutes les sociétés humaines, les phénomènes économiques furent insérés dans les autres institutions sociales, l'économie étant pensée et gérée en tant qu'auxiliaire de la société dans laquelle elle est encastrée: mais au nom du mythe du marché autorégulé, nous aboutissons au contraire: la société est considérée à l'aune du marché, l'économie est désencastrée des relations sociales et une référence autonome juridique aux « êtres humains »a disparu « de la liste des objectifs assignés à l'économie ».

\footnotetext{
${ }^{26}$ NADEAU Gilles, film France 1999, « Keynes ou le capitalisme sous antidépresseurs 》 http://www.dailymotion.com/video/xaoxdt_keynes-ou-le-capitalisme-sous-antid_news,

27 HAYEK Friedrich, Droit, législation et liberté (1973-1979) (Law, Legislation and Liberty) est le magnum opus du «Prix Nobel » (en 1974) d'économie et philosophe libéral Friedrich Hayek.

${ }^{28}$ LE MOIGNE Jean-Louis « Auto-éco-ré-organisation sociale et complexité : des desseins humains pour et par l'action humaine » (Archive), écrit dans le cadre du Colloque Cerisy 99 « Hayek et la Philosophie économique », 1999. http://lemoigne.unblog.fr

${ }_{29}^{29}$ CAILLE Alain, Splendeurs et misères des sciences sociales. Esquisse d'une mythologie, Droz, 1986.

${ }^{30}$ HILLENKAMP Isabelle, LAVILLE Jean-Louis, Socio économie et démocratie, l'actualité de Karl Polanyi, Editions érès, Toulouse, 2013.
} 
Pour lui, l'aboutissement de ce double mouvement de marchandisation du monde et de réductionnisme individualiste, maximalise l'idée d'autonomie absolue qui sape les bases du 'vivre ensemble' nécessaire à toute société.

Miguel BENASAYAG ${ }^{31}$ va dénoncer le mythe de l'individu autonome: « La société de l'individu est d'emblée la société de la séparation, société de sujets potentiellement désincarnés qui se situent face au monde et au réel. »

Comment cette idéologie ou pensée unique va t'elle s'incarner au niveau politique et économique.

Edouard DELRUELLE ${ }^{32}$, nous dit : « dans la logique néolibérale, l'Etat lui-même doit être concu comme une entreprise d'un type particulier - une entreprise au service des entreprises, qui doit lutter contre les rigidités, favoriser la flexibilité des acteurs, les préparer à la concurrence, et surtout les protéger contre toutes les formes d'atteinte à la propriété et à la performance : 'bureaucratie, assistanat, gréviculture'... "

Le néo-libéralisme aliène les hommes réduits à des «homo oeconomicus» exclusivement. Le cœur stratégique des politiques néo-libérales génère un individualisme exacerbé et la 'fabrique de l'homme endetté' (LAZZARATO) ${ }^{33}$, il s'agit de réduire toute la société elle même à une société de marché. Et l'homme devient lui-aussi entrepreneur de sa propre vie

"HAYEK ${ }^{34}$ arrache le thème du progrès aux socialistes. régle du marché absolue , selection naturelle / justice sociale illusoire/. C'était son obsession: faire du néolibéralisme une philosophie progressiste, montrer que les véritables conservateurs sont ceux qui croient en la justice sociale et qui de ce fait, retombent dans l'illusion constructiviste des sociétés arriérées, où le pouvoir souverain est censé tout maîtriser à partir de principes à priori. Quant à l'égalité, qui nie les différences d'aptitude et de performance, elle ne peut déboucher que sur la dictature du conformisme et de la médiocrité. Le socialisme sous toutes ses formes est ringardisé. Sur ce plan la victoire idéologique de Hayek est totale : aujourd'hui c'est bel et bien la droite qui passe pour le parti des réformes et de la modernisation et la gauche pour celui des droits acquis et des rigidités administratives »

\section{$\rightarrow$ Marchandisation du travail social}

Dans une publication du CDGAI ${ }^{35}$ \& de l'Observatoire ${ }^{36}$ je dénonce les dérives du néo libéralisme pour la sphère du Travail Social : " l'intégration européenne s'est construite sur le dogme de la pensée néolibérale ...La crise économique des années 1970 met en

\footnotetext{
${ }^{31}$ BENASAYAg Miguel, Le mythe de l'individu, p.22, Editions La Découverte, Paris, 2004.

${ }^{32}$ DELRUELLE Edouard, De l'homme et du citoyen, de boeck, p. 181, 2014.

${ }^{33}$ LAZZARATO Maurizio: « La Fabrique de I'homme endetté. Essai sur la condition néolibérale» Editions Amsterdam, 125 pages, http://www.lemonde.fr/idees/article/2011/09/26/la-fabrique-de-l-homme-endette-demaurizio-lazzarato_1577745_3232.htm|\#kBApuC2clHYGVWdH.99

${ }^{34}$ HAYEK Friedrich ( 1899-1992) philosophe et économiste britannique originaire d'Autriche, prix Nobel d'économie en 1974.

${ }^{35}$ DONJEAN Christine, «Le travail social d'aujourd'hui est-il devenu barbare », p.p.9-10, Collection Travail en action, CDGAI, Liège, 2015, réflexion-débat autour du livre de VRANCKEN Didier, le Social barbare, Collection Question de société, juin 2010, http://media.wix.com/ugd/08846d_ec0745f2da734cfcaf332ac77cf74f3b.pdf

${ }^{36}$ www.actionsocialeendanger.be collectif, « Autonomie \& Travail Social, de l'espoir au désenchantement» dans L'OBSERVATOIRE, L'autonomie en tension, octobre 2016, 
question le modèle des États-providence....dans ce contexte, la dérégulation ${ }^{37}$ se traduit par une réduction des interventions de l'Etat dans l'économie, mais aussi dans le champ du social, par la privatisation d'entreprises publiques, I'abandon ou l'assouplissement de réglementations....Cette dérégulation ... voit émerger la marchandisation du Travail Social. » Un phénomène typique de contagion s'opère : l'extension, d'une vision de plus en plus large de ce que recouvre la notion de « service d'intérêt économique général », pour venir maintenant englober les « services sociaux d'intérêt général ». 38 \& 39

L'Etat, sous l'égide des directives européennes, devient 'social actif', le travail social, éducatif, culturel,... eux aussi sont ainsi appelés à épouser les mêmes méthodes et les mêmes contraintes que les champs couverts par l'économie de marché. C'est donc bien une tentative presque aboutie pour faire passer l'économie de marché vers 'la Société de Marché ', où le social est désormais vu d'avantage comme un coût qu'il convient de réduire que comme un investissement en faveur de la justice sociale et du bien-être collectif.

\section{Grille d'interprétation du monde}

\section{$\rightarrow$ Théorie darwinienne de l'évolution au centre de l'analyse des organisations}

La main invisible de Adam Smith est remplacée par la théorie de l'évolution de DARWIN. ...le marché ce n'est pas l'échange mais la concurrence.

Certains chercheurs en organisation trouvent que la théorie de la contingence accorde trop d'importance à la souplesse et au pouvoir des acteurs de l'organisation, et pas assez à l'environnement de celle-ci, force qui va être déterminante dans sa survie ou sa mort. Ils réinterprètent la théorie de la contingence en gommant le rôle des Acteurs internes à l'organisation au profit des forces externes.

"Plus la société est ouverte, plus elle produit de complexité, donc requiert plus de sélection naturelle, et plus elle s'adapte, se développe et progresse. "

$\mathrm{Ce}$ qui semble clair ici, c'est qu'ils renversent simplement la causalité qui va donc être linéaire, de l'environnement (presque exclusivement regardé sous son angle du « marché » et de la « finance», l'écosystème étant évacué ...) vers les organisations qui devraient se reconfigurer autrement pour survivre.

La volonté humaine et notamment les valeurs éthiques qui l'accompagnent sont escamotées. La survie écologique de la planète également.

On retrouve donc certains aspects de ces théories dans le «néolibéralisme» nous en

\footnotetext{
${ }^{37}$ La dérégulation désigne une politique qui consiste à réduire ou à supprimer la régulation d'un secteur économique ou d'une profession, ou bien à rendre plus libre la fixation des prix. Elle a pour objectif de favoriser la concurrence et l'innovation en s'appuyant sur le postulat d'une dynamique naturelle des marchés en question et de leur capacité d'autorégulation

${ }^{38}$ Cahiers de la Solidarité $n^{\circ} 27$ : « Services sociaux d'intérêt général : entre finalité sociale et libre-concurrence » http://www.uniopss.asso.fr/resources/trco/pdfs/2011/C_mars_2011/60173_CahierSolidarite27_SSIG_web.pdf

${ }^{39}$ HENRY Joël, Intervention à CNAUMO Eurocef/Conseil de l'Europe) dans le cadre du colloque "Le travail social à l'épreuve du management et des impératifs gestionnaires" organisé par l'Université Toulouse II-Le Mirail http://www.canalu.tv/video/universite_toulouse_ii_le_mirail/l_europe_le_travail_social_et_les_ssig_services_sociaux_ d_interet_general.5266
} 
pointons cet étrange paradoxe, puisque la concurrence est le résultat de conditions qui auront soigneusement et artificiellement été aménagées...par l'Etat lui-même.

\section{$\rightarrow$ MYTHE des Technocraties vécues comme « compétentes »}

Mais la définition de la democratie, du rôle de l'ETAT et de ses institutions, n'est pas confisquée que par les financiers ou les capitalistes et les politiques « ultra libéraux » Alors que le renforcement de l'État depuis le début du XIXème siècle a contribué à accroître le rôle du gouvernement et de l'administration, le pouvoir technocratique, dans les États de la fin du XXe siècle, a lui plutôt tendance à se substituer aux élus.

Des légions de technocrates envahissent les bureaucraties elles-mêmes ou ils se trouvent en technostructure par rapport à celles-ci

Les experts détiennent une place toujours plus grande dans le processus de décision et développent un réseau de relations avec les groupes pour dégager des compromis. La négociation contractuelle risque ainsi de remplacer systématiquement la délibération démocratique, traditionnellement appelée à réguler les intérêts conflictuels.

Les exemples récents du CETA, mais aussi des autres traités commerciaux en négociation (TTIP) sont éclairants à ce sujet. Les négociations entre Experts, escamotent la place des politiques.

Dans le processus actuel de mondialisation, les experts sont omniprésents et leurs propres décisions dépendent de plus en plus de facteurs exogènes : situation du marché international, flux des investissements étrangers, mouvements des capitaux. politiques économiques orchestrées par la troika (Fonds monétaire international, Banque mondiale, Commssion européenne,), instance internationale non élue.

Cette technocratie est parfois inféodée à ce monde extérieur qui finance les expertises De nombreux «lanceurs d'alerte» dénoncent ces collusions. Les liens entre monde des multinationales et son réseau de lobbyistes (plus de 500 aux alentours de Bruxelles), technocrates et politiques sont ici bouleversants.

Comment ces collusions passent-elles quasiment inaperçues? Les progrès technologiques mondiaux imposent donc des décisions fondamentales pour le développement industriel du pays, avec des expertises de plus en plus fines et complexes elles-mêmes .

La «technicisation» de la société réduit considérablement l'éventail des décisions que peut prendre le citoyen lambda. Qui se sent alors incompétents face à ses experts hyper spécialisés.

Mais ce rapport avec la connaissance n'est il pas plus ambigü? selon Jacques GODBOUT ${ }^{40}$ dans Ethique Publique

"La technocratie est l'une de ces tentations permanentes de la démocratie visant à identifier le méta-niveau au mythe dominant actuellement la société, origine et fondement de la vérité: la Science.

si les décisions étaient fondées sur la science (et sur la compétence qui permet à certains seulement d'y accéder), on mettrait fin à cette boucle gênante qui ramène tout

\footnotetext{
${ }^{40}$ GODBOUT Jacques T. « Pas de représentation sans représentativité ?», Ethique publique, Vo. $7, n^{\circ} 1$, Nouvelles formes de démocratie, 2005, https://ethiquepublique.revues.org/1894
} 
à l'ignorant homme de la rue. L'idéologie technocratique est cette tentation permanente de la démocratie de se trouver un méta-niveau inviolable comme dans le bon vieux temps, de remplacer le Roi et Dieu par la Science, de ne pas vraiment assumer l'état " désenchanté » du monde, dirait Weber. On réintroduit ainsi la hiérarchie linéaire sans possibilités de boucle étrange, en tentant en permanence de transformer les rapports gouvernants-gouvernés en rapports de feedback, le niveau inférieur devant alors se contenter de 'nourrir ' les niveaux supérieurs. Le peuple est alors réduit au seul statut de niveau inférieur."

Mais si les technocrates se réfèrent eux-mêmes à une autorité externe, ce sont bien les lois de la finance et du marché qui dictent les règles du jeu et l'Etat doit s'organiser pour baisser la fiscalité, les protections sociales et les rigidités administratives. Les hommes politiques sont délégitimés et ils ne savent plus imposer aujourd'hui leurs volontés aux acteurs financiers \& économiques (impuissances, soumissions parfois collusions et/ou corruptions), il leur est demandé une orchestration dans le cadre des gouvernements: soutenir l'économie par les lois, par l'élaboration de normes sociales qui faciliteront la concurrence, le libre-échange et l'action économique de tous les acteurs et de toutes les institutions sociales.

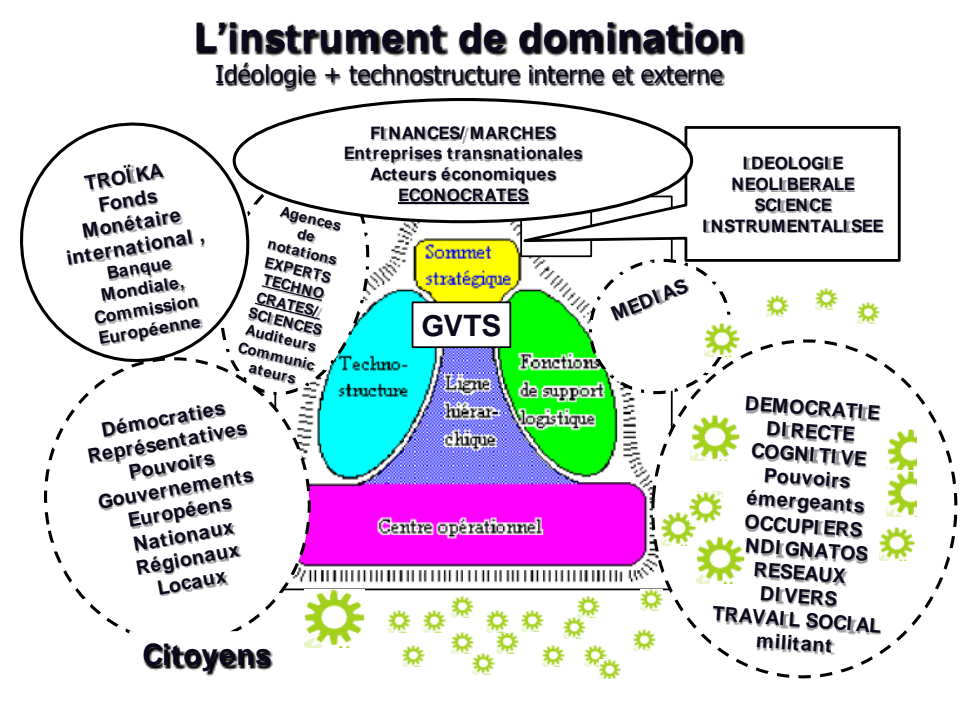

\section{Quelles intentions et visions du futur du/des modélisateur(s) ???}

\section{$\rightarrow$ Pouvoir pervers ou « soft tyrannie »}

Que le pouvoir soit inféodé à l'idéologie « Néolibérale» ou à « la technocratie » (sous le couvert de la vérité de la science), le programme officiel reste le plus souvent celui de la « participation citoyenne» qui n'est qu'un simulacre. Cela s'accompagne d'une crise de légitimité des institutions politiques, donc tant de la démocratie représentative que délibérative.

Ainsi donc, le plus souvent, les acteurs de la base sont pris dans des «doubles liens» sociétaux et institutionnels. 
C'est une même sorte de 'barbarie douce' que l'on trouve dans le champ du social sous la forme de la gouvernance terme inventé pour le modèle gestionnaire ou new management. On y parle de rentabilité, d'efficacité, d'efficience, de rationalisation, d'actions à court terme, de cofinancements publics/privés, de mise en concurrence dans le cadre d'appels d'offres, d'évaluation, etc. Les logiques de rationalisation s'appliquent désormais aux usagers, mais aussi aux managers des institutions et aux travailleurs sociaux euxmêmes. ${ }^{41}$

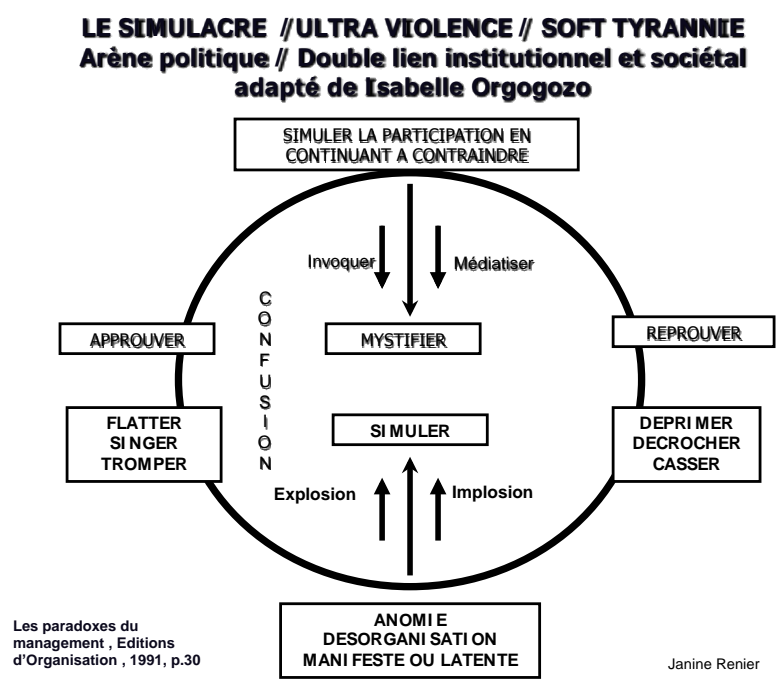

$\rightarrow$ MYTHE du Chef, vécu comme « Sauveur Providentiel »

Le risque est grand qu'en période de crise, ce « déficit démocratique » ne soit comblé par une plus grande personnalisation du pouvoir et par la renaissance du mythe du chef salvateur auréolé d'incantations « démagogiques»qui fleurissent un peu partout.

L'exercice politique se porte le mieux lorsque l'équilibre des pouvoirs est réalisé. Le risque politique réside dans la concentration des pouvoirs, visant à une sacralisation d'un "Chef éclairé », éliminant ou asservissant tout ce qui, d'une façon ou d'une autre, amoindrirait cette autorité. La place prééminente du chef de l'État, la suprématie de la décision politique sur toute forme de droit, est alors renforcée par une mécanique démagogique, qui peut en effet laisser craindre de sérieuses dérives, l'histoire en ayant régulièrement été témoin, sous différentes formes. Le recul des « Droits de l'homme » et de la recherche de la « justice sociale» peut enclencher toute notre inquiétude!!

\section{3) 4 - CERVEAU \\ VERS LA PENSEE DE LA COMPLEXITE}

\section{Contexte socio-politique, économique, éthique}

Hölderlin : « Là où croît le péril, croît aussi ce qui sauve. »

41 ORGOGOZO Isabelle: les paradoxes du management, P30 „, Les EDITIONS D'ORGANISATION, 1991 
$\rightarrow$ Deux barbaries différentes nous menacent. ${ }^{42}$

"La première, ancienne, est charriée par toute l'histoire humaine: c'est la haine, le mépris, le meurtre, la torture. Naivement, nous l'avons cru éliminée avec la fin du nazisme et du stalinisme. Mais au début des années 1990 ont éclaté les guerres de religions, notamment en ex-Yougoslavie.

Aujourd'hui, cette barbarie archaïque s'incarne surtout dans une organisation criminelle, Daech, qui nous menace sur notre propre sol.

A nouveau, les formes agressives de la religion et de l'ethnie suscitent ou nourrissent des conflits. (SYRIE)

L'autre barbarie, glacée, froide, est l'hégémonie du profit, du calcul et de l'anonymat. Les milieux officiels croient tout connaître par le chiffre, par le taux de croissance, par le PIB et les sondages d'opinion... Or les chiffres restent à la surface de nos réalités humaines. Aucun ne mesure la souffrance, le bonheur ou le malheur. »

\section{Grille d'interprétation du monde}

\section{$\rightarrow$ Un des premiers remèdes : la démocratie cognitive}

« La dépossession du savoir, très mal compensée par la vulgarisation médiatique, pose le problème historique clé de la démocratie cognitive. La continuation du processus technoscientifique actuel, processus du reste aveugle qui échappe à la conscience et à la volonté des scientifiques eux-mêmes, conduit à une régression forte de démocratie. Il n'y a pas pour cela de politique immédiate à mettre en oeuvre. Il y a la nécessité d'une prise de conscience politique de l'urgence à oeuvrer pour une démocratie cognitive....

Il est effectivement impossible de démocratiser un savoir cloisonné et ésotérisé par nature. Mais il est de plus en plus possible d'envisager une réforme de pensée qui permettrait d'affronter le formidable défi qui nous enferme dans l'alternative suivante: ou bien subir le bombardement d'innombrables informations qui nous arrivent en pluie quotidiennement par les journaux, radios, télévisions, ou bien nous confier à des systèmes de pensée qui ne retiennent des informations que ce qui les confirme ou leur est intelligible, rejetant comme erreur ou illusion tout ce qui les dément ou leur est incompréhensible. Ce problème se pose non seulement pour la connaissance du monde au jour le jour, mais aussi pour la connaissance de toutes choses sociales et pour la connaissance scientifique elle-même. »

La question étant dés lors, comment participer à sa mise en place, nous esquisserons des pistes possibles au point 5.

$\rightarrow$ Le paradigme de la complexité

"Tout système vivant (auto organisé), même le plus simple, combine un très grand nombre d'unités (cellules, molécules). Plusieurs complexités s'entrecroisent (microphysique, macro-physique, biologique, psychique, sociale, économique, écologique)

\footnotetext{
${ }^{42}$ KERCHOUCHE DALIDA, dans le Figaro Madame, interview de Edgar Morin: "Nous traversons une crise profonde de civilisation", 11 septembre 2016
} 
La terme complexité vient de complexus 'ce qui est tissé ensemble' cela stipule un très grand nombre de niveaux hiérarchiques, un très grand nombre de constituants différents, une extrême quantité d'interactions et d'interférences.

Penser la complexité revêt de multiples défis: rencontrer l'incertitude, l'imprévisibilité, la diversité, la contradiction. Dans un sens, elle a toujours affaire avec le hasard

Si la complexité est non pas la clé du monde, mais le défi à affronter, la pensée complexe est non pas ce qui évite ou supprime le défi, mais ce qui aide à le relever, et parfois même à le surmonter» 43

\section{COMPLEXITE $=$ complexus = ce qui est tissé ensemble}

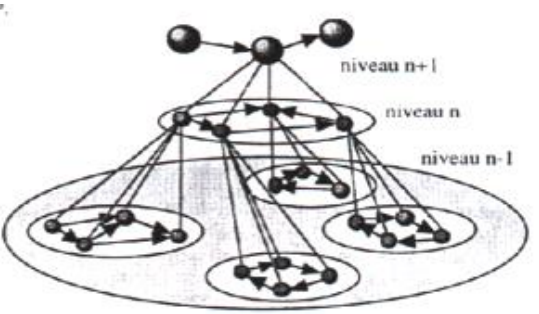

- Ouverture sur I' environnement multiforme

- Incertitudes Indéterminations

- Hasard et imprévisibilitế

- Très grand nombre de niveaux hiếrarchiques

- Très grand nombre de constituants différents - Extrême quantité d' interactions

"Complexité pour décloisonner les consciences, maîtriser les peurs, faire s'entrappeler les idéaux, métisser les imaginations, et ainsi "ré enchanter l'espérance" cultivée dans la fraternité, la solidarité et l'exaucement de sens. La liberté et l'égalité peuvent s'imposer par la loi ; pas la fraternité. Elle doit se construire, s'éduquer, venir de l'intérieur des citoyens éclairés sur les enjeux et les mécanismes des différents pouvoirs. »

La pensée de la complexité se présente comme une sorte d'édifice à plusieurs étages. La base est formée à partir de trois théories (information, cybernétique et système) et comporte les outils nécessaires pour une théorie de l'organisation. Vient ensuite un deuxième étage avec les idées de l'auto-organisation.

A cet édifice, Edgar MORIN a voulu apporter des éléments supplémentaires. Notamment trois principes ${ }^{44}$ \& qui vont venir illustrer ce concept, partons des écrits de Morin lui-même, et également ceux de Dominique GENELOT ${ }^{45}$

\section{1/- Le principe hologrammatique}

En 1948, Dennis GABOR, utilise un appareil de photo sans lentille pour enregistrer l'information d'une façon qui emmagasine le tout dans chacune de ses parties. Une des

\footnotetext{
${ }^{43}$ MORIN Edgar, Introduction à la pensée complexe, Paris, E.S.F., collection communication et complexité, 1990

${ }^{44}$ LE MOIGNE Jean-Louis, MORIN Edgar: L'intelligence de la complexité, l'Harmattan, 1999.

${ }^{45}$ GENELOT Dominique: Manager dans le complexité, réflexion à l'égard des dirigeants, INSEP CONSULTING Editions, 4eme édition, Paris 2011 
caractéristiques de l'hologramme est que, s'il se brise, n'importe laquelle de ses parties peut-être utilisée pour reconstruire l'image entière.

"Le principe hologrammatique met en évidence cet apparent paradoxe de certains systèmes, où non seulement la partie est dans le tout, mais où le tout est dans la partie. Ainsi, chaque cellule est une partie d'un tout - l'organisme global - mais le tout est également dans la partie : la totalité du patrimoine génétique est présente dans chaque cellule individuelle. »

De la même façon, l'individu est une partie de la société, mais la société est présente dans chaque individu en temps que tout à travers son langage, sa culture, ses normes et sa participation active à la vie citoyenne et publique:

\section{2/- Le principe de récursion organisationnelle}

Ce concept est complémentaire du précédent.

Le tout et les parties sont liés dans une dialectique dynamique, les interactions sont inextricablement enchevêtrées.

Dans un processus récursif, les effets produits sont nécessaires au processus qui les génère.

«Ce principe va au delà du principe de rétroaction (feed-back); il dépasse la notion de régulation pour celle d'autoproduction et d'auto-organisation. Cette boucle génératrice dans laquelle les produits et les effets sont eux-mêmes créateurs de ce qui les produit. Ainsi, nous individus, sommes les produits d'un système de reproduction issu du fond des âges, mais ce système ne peut se reproduire que si nous-mêmes nous en devenons les producteurs en nous accouplant.

Les individus humains produisent la société dans et par leurs interactions, mais la société, en tant que tout émergeant, produit l'humanité de ces individus en leur apportant le langage et la culture »

La société, à travers la famille et diverses autres institutions (dont l'école, et les institutions démocratiques), façonne l'individu qui est lui-même le constituant de base et sujet /acteur/auteur /générateur de la société.

La spirale récursive est ainsi enclenchée.

Dans un registre plus existentiel, l'homme est lui-même une boucle de récursivité : son intelligence lui permet d'apprendre, de progresser, cette connaissance développe son intelligence, participe de sa transformation et la boucle récursive du développement humain se poursuit.

Dans la réalité, les interactions ne sont jamais linéaires et très claires. Les niveaux sont inter-reliés et les événements se déroulent en cascades et font parfois un long détour dans l'espace et le temps, avant de se reboucler sur les faits qui les avaient déclenchés. Il faudrait parler de causalités et d'interactions récursives, spirales ou tourbillonnaires. Dans l'espace démocratique, l'on parlera tantôt hiérarchies enchevêtrées, tantôt de hiérarchie bouclée démocratique de Russell Lincoln ACKOFF tantôt encore de boucles étranges de D. HOFSTADTER, profonds changements dans nos représentations ....des solidarités autonomisantes et des autonomies solidarisantes. 


\section{3/- Le principe dialogique}

"Il unit deux principes ou deux notions antagonistes, qui apparemment devraient se repousser l'une l'autre, mais qui sont indissociables et indispensables pour comprendre une même réalité.

La pensée humaine impose aux perceptions comme aux discours l'alternative logique du vrai et du faux. C'est avec le langage qu'apparaît et fonctionne sans trêve le couperet logique inexorable de la négation»

\section{Dialogique \& Démocratie}

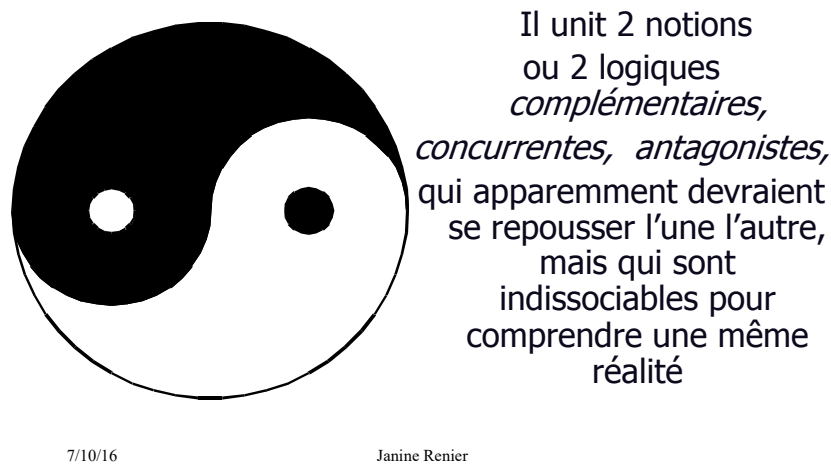

Le vivant est un processus paradoxal qui se structure ${ }^{46}$ (BAREL), des logiques différentes qui peuvent être à la fois complémentaires, concurrentes, voire antagonistes, se trouvent réunies et coexistent dans une même unité, sans que leurs différences ne soient pour autant gommées par cette unité. C'est ce qui précisément va rendre le processus vivant.

- Sujet/Objet

- Ordre/désordre

- Qualité / Quantité

- Sentiment / Raison

- Liberté / Déterminisme

- Autonomie/ hétéronomie

- Individuel/ collectif

- Logique/analogique

- Economique/social

- Directivité /délégation

- Paix / Violence

- Humanité / barbarie

- Autonomie /Solidarité

- Individuel / Collectif

- Certitude / incertitude

\footnotetext{
${ }^{46}$ BAREL Yves: Le paradoxe et le système: essai sur le fantastique social, [suivi de Le paradoxe dix ans après], presses universitaires de Grenoble, 1989. 
- Maîtrise / Lâcher prise

- Sécurité / Peur

- Vie / Mort

- Harmonie / Dysharmonie

- Culture humaniste / Culture scientifique

"Dans chaque cas, l'existence d'un terme dépend de l'existence de l'autre, nous ne pouvons savoir ce qu'est le froid si nous ignorons ce qu'est le chaud, nous ne pouvons concevoir le jour si nous ne connaissons pas la nuit. Le bien définit le mal et la vie définit la mort. Les contraires s'entrelacent dans un état de tension qui définit aussi un état d'harmonie et de complétude. "47

La coexistence de logiques différentes est un phénomène extrêmement fréquent, caractéristique des situations du vivant et des situations complexes.

Le processus de "disjonction-exclusion 》 est destructeur

Le processus de « distinction-conjonction » est créateur

\section{Quelles intentions et visions du futur du/des modélisateur(s) ???}

\section{$\rightarrow$ Vers une auto-éco-organisation}

Edgar MORIN ${ }^{48}$ va finir l'édifice en enrichissant le concept d'auto organisation par celui d'auto-éco-organisation: l'autonomie des organismes vivants est inséparable de leur dépendance à l'égard de leur environnement.

"Ici apparaît dès lors le point le plus crucial de la nouvelle notion d'autonomie; un système ouvert est un système qui peut nourrir son autonomie mais à travers la dépendance à l'égard du milieu extérieur. Cela veut dire que, contrairement à l'opposition simplifiante entre une autonomie sans dépendance et un déterminisme de dépendance sans autonomie, nous voyons que la notion d'autonomie ne peut être conçue qu'en relation avec l'idée de dépendance. Ce paradoxe fondamental est invisible à toutes les visions dissociatrices pour qui il y a antinomie absolue entre dépendance et indépendance. C'est cette pensée clef d'autonomie/dépendance que la réalité nous oblige à concevoir. Et du reste, plus un système développera sa complexité, plus il pourra développer son autonomie, plus il aura des dépendances multiples. Nous-mêmes, nous construisons notre autonomie psychologique, individuelle, personnelle, à travers des dépendances que nous avons subies qui sont celles de la famille, la dure dépendance au sein de l'école, les dépendances au sein de l'Université. Toute vie humaine autonome est un tissu de dépendances incroyables. Bien entendu, si ce dont nous dépendons nous manque, nous

\footnotetext{
${ }^{47}$ GENELOT Dominique: Manager dans le complexité, réflexion à l'égard des dirigeants, INSEP CONSULTING Editions, 4eme édition, Paris 2011.

${ }^{48}$ MORIN Edgar, «Peut-on concevoir une science de l'autonomie ? » dans l'Auto-organisation, colloque de Cerisy, Editions du Seuil, 1983, pp.317-325 
sommes perdus, nous sommes morts; cela veut dire aussi que le concept d'autonomie est un concept non substantiel mais relatif et relationnel. Je ne dis pas que plus on est dépendant, plus on est autonome. Il n'y a pas de réciprocité entre ces termes. Je dis qu'on ne peut pas concevoir d'autonomie sans dépendance ».

"Selon cette conception, l'autonomie ne signifie pas 'suppression des dépendances' puisque, à l'intérieur d'un système, on ne peut être autonome que par ou à travers des dépendances. ... Il faut donc considérer l'autonomie de l'individu dans le paradoxe de sa dépendance à l'égard des gênes (auto-dépendance)... et à l'égard de l'empire de l'environnement (éco-dépendance). L'accolement des termes antagonistes autonomieldépendance, différence lappartenance suggère que l'individu acquiert son autonomie dans et par les dépendances, les servitudes pour cesser d'être le pur jouet des aléas. »

\section{$\rightarrow$ Pouvoir ascendant ou Empowerment véritable ?}

L'autonomisation des citoyens se construira donc à partir du repérage des aliénations qui pèsent sur eux et de leur positionnement par rapport à celles-ci, c'est donc bien à partir de ce repérage que se dégagera un certain degré de liberté. Et celui-ci nécessite bien une « démocratie cognitive», à l'école ${ }^{49}$, dans les universités citoyennes, les débats délibératifs, les réseaux de résistance engagés. Etc.

La conception du pouvoir et de l'autorité est aussi radicalement changée, d'exclusivement verticale, elle est aussi devenue horizontale ${ }^{50}$

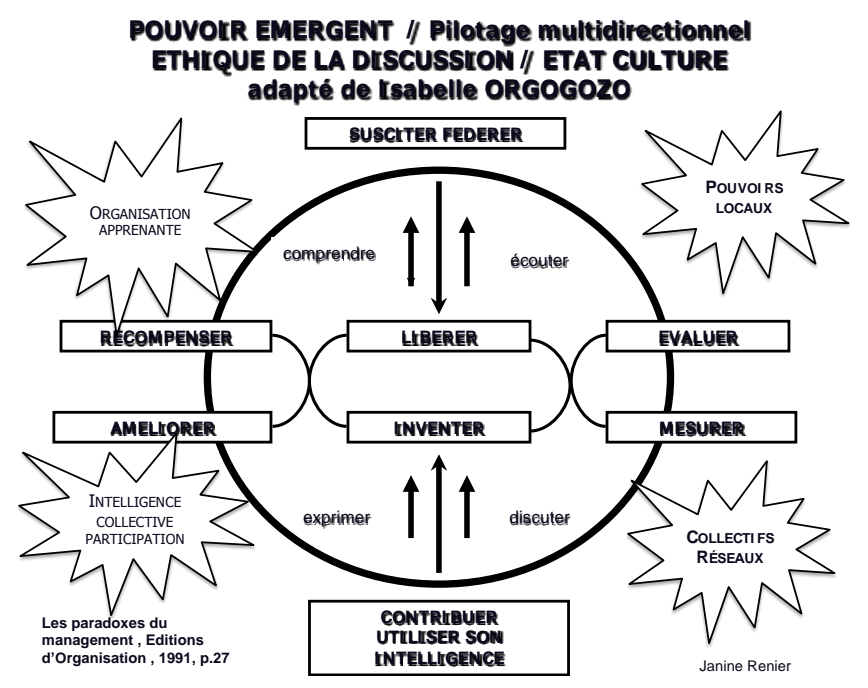

\section{$\rightarrow$ Vers une « écologie de l'action »}

Edgar MORIN a aussi campé dès 1980 ce phénomène en termes stratégiques et politiques, sous le nom imagé d'écologie de l'action:' toute action échappe à la volonté de son auteur en entrant dans le jeu des inter-rétro-actions du milieu où elle intervient.

\footnotetext{
49 MORIN EDGAR, «Les 7 savoirs nécessaires à l'éducation du Futur », Publié en octobre 1999 par l' organisation des Nations Unies pour l'éducation, la science et la culture - 7 place de Fontenoy - 75352 Paris 07 SP - France UNESCO1999 ,http://unesdoc.unesco.org/images/0011/001177/117740fo.pdf

50 ORGOGOZO Isabelle: les paradoxes du management, P.27, Les EDITIONS D'ORGANISATION, 1991 
Tel est le principe propre à l'écologie de l'action ... L'écologie de l'action c'est en somme tenir compte de la complexité qu'elle suppose, c'est-à-dire aléa, hasard, initiative, décision, inattendu, imprévu, conscience des dérives et des transformations ...'

Ceci donc va modifier de manière notable la vision du pouvoir et de la gestion: la planification qui ne pourra plus être stratégique, ni linéaire, ni même exclusivement descendante, mais qui devra être vue comme un processus évolutif, se transformant au fil du temps, avec des acteurs et des environnements multiples, évoluant eux-mêmes et qui seront au non imprégnées de valeurs éthiques transcendantes ????

Ce concept devrait à lui être susceptible d'expliquer en partie pourquoi les hommes politiques éprouvent tant de mal à « tenir leurs promesses», puisque la plupart du temps, ils réalisent leurs prévisions comme de hommes providentiels et des sauveurs en termes de 'programmations linéaires', en isolant l'état nation de ces multiples interdépendances ... Les promesses sont plus simples à élaborer et les enjeux de souveraineté, d'émancipation, de lutte contre l'aliénation économique et culturelle, sont souvent éludés ... ou tus ....

\section{3) 5 - CULTURE \\ VERS UNE ETHIQUE DE RELIANCE}

\section{Contexte socio culturel, socio-politique, économique, éthique}

\section{$\rightarrow$ Liens entre CULTURE \& MYTHES}

« La culture ne peut être objectivée, c'est en participant à son mythe qu'il est possible d'approcher et connaître une culture donnée. Chaque culture est une galaxie comprenant une expérience et une perception du monde d'où émerge sa propre compréhension, qui lui donne son originalité et qui détermine ce qui est significatif pour une collectivité : les critères de vérité, de bonté et de beauté, ainsi que les limites du monde et la façon d'y être. «PANIKKAR Raimon ${ }^{11}$

La modernité nous a révéler 3 grands mythes: celui de la raison absolue, du progrès infini et du bonheur total avec au plan démocratique l'universalisation des intérêts.

Les mythes fondateurs de la démocratie: liberté, égalité, fraternité /solidarité, ainsi que les droits de l'homme préludent à sa naissance.

Bouclons donc ceci avec notre raisonnement de départ et sommes nous à la fin de I'histoire? (Démocratie parlementaire et économie de marché), comme le disait Francis FUKUYAMA, alors que sévit une véritable crise de la civilisation qui menace tout notre système : atteinte écologique, malaise économique, système médiatique déchaîné et culture en pleine transformation. La démocratie représentative ne disposerait plus en termes organisationnels les capacités de rencontrer cette complexité exponentielle,

\footnotetext{
${ }^{51}$ PANIKKAR Raimon, " Cultures et dialogue interculturel " Forum Universel des Cultures 2004
} 
tant à l'interne que à l'externe, nous l'aborderons dans les points suivants. A la fois, dans la démocratie délibérative sous tendue par la démocratie cognitive

\section{Grille d'interprétation du monde}

\section{$\rightarrow$ Changement de paradigme dans la manière de faire de la politique}

La démocratie vue comme un ensemble de formes de vies dont découle la solidarité ou la démocratie vue bien plus comme une forme de société que comme un régime politique.

Selon I'historien Pierre ROSANVALLON, ${ }^{52}$ « la démocratie est également une forme sociale, dans ce sens elle se lie au concept d'égalité».

De cette conception découle la question suivante: dans quelle société souhaite-t-on vivre? La démocratie ne peut pas se concevoir uniquement comme un régime politique, elle repose également sur des formes de solidarité sociale sans lesquelles ni la démocratie ni l'égalité ni les libertés publiques ne peuvent exister.

L'égalité, conçue comme un ensemble de droits fondamentaux, est un mot d'ordre politique qui ne prend sens que s'il est ancré dans des formes de solidarité concrètes.

Si les formes de solidarité sociale sont conçues comme le ciment de la démocratie, elles permettront de donner une existence autre que juridique aux droits fondamentaux.

Or la situation actuelle étant marquée par les tensions croissantes sur les ressources naturelles, l'accroissement des inégalités et de la précarité, l'instabilité économique est porteuse de risques de désolidarisation de nos sociétés car elle remet en cause les formes de solidarité sociale.

La représentation verticale du pouvoir a changé vers un mode plus horizontal mais plus qu'un élément de réflexion philosophique, plus qu'une mode, l'éthique est devenue une condition de succès du fonctionnement d'une saine démocratie... Si le comportement éthique est d'abord et avant tout une responsabilité personnelle appuyée sur des valeurs d'honnêteté, de droiture et de justice, le développement d'une culture éthique forte doit relever elle-même d'une co-construction en reliance avec d'autres.

\section{$\rightarrow$ Vers une PRAXEOLOGIE}

Elle contribuera à son tour à la consolidation de la démocratie, avec les droits et libertés qu'elle apporte. Ce processus s'incarnera dans une mise en actes, véritable «praxéologie»

"L'éthique vue comme une science de l'action juste ne se réduit pas à des mots, des indications formelles, générales, abstraites ${ }^{53}$."

Elle peut éclore dans des structures «ad hoc » (souvent des réseaux), se développer dans la coopération conflictuelle, s'incarner dans des actions équitables, jointes à la

\footnotetext{
52 GODMER Laurent, SMADJA David, «Entretien avec Pierre ROSANVALLON « dans Raison Politiques, https://www.cairn.info/publications-de-\%20Godmer-Laurent--78966.htm ,PP 173 - 199.

${ }^{53}$ VANOTTI, M., Le silence comme un cri à l'envers, maltraitance et abus sexuels envers les enfants, Edition Médecine et Hygiène 1992 
co-construction des savoirs qui va s'opérer dans la démocratie cognitive.

Celle-ci sera essentiellement possible, dans un premier temps, dans les projets, à l'échelle locale ainsi que nous l'illustrerons dans le plans de cohésion sociale. Changement qui ne pourrait prendre ses racines que dans une nouvelle épistémologie de la complexité en lien avec la démocratie cognitive ${ }^{54}$ qu' Edgar Morin appelle de ses vœux...où les débats seraient aussi portés par les citoyens.

\section{$\rightarrow$ Les théories de l'Ethique, Evolution au fil du temps}

\section{1/LES ETHIQUES DE LA RESPONSABILITE : conviction/responsabilité}

- Max WEBER (1864 - 1920),

- Hans JONAS (1903 - 1993)

Dans le domaine de la politique et de l'action sociale, WEBER ${ }^{55}(1864$ - 1920) oppose l'éthique de conviction (qui ne se préoccupe que du principe moral présidant à l'action sans se soucier des conséquences) et l'éthique de responsabilité (selon laquelle les conséquences sont prises en compte).

À ceux qu'attire la sphère sociopolitique, il demandait d'être mus à la fois par l'éthique de conviction et par l'éthique de responsabilité, qui accepte de prendre conscience des risques qu'entraîne logiquement toute décision et s'appuie sur une estimation raisonnée des conséquences prévisibles.

Ici, nous voyons donc bien la force des concepts de multirationalité et de réticorationalité, opérante dans les reseaux, par rapport au concept de rationalité limitée des bureaucraties.

François RAYNAUD ${ }^{56}$ résume la position de WEBER de la façon suivante : « Là où le partisan de l'éthique de conviction ne se sent responsable que de la nécessité de veiller sur la flamme de la pure doctrine afin qu'elle ne s'éteigne pas, le partisan de l'éthique de la responsabilité estime au contraire impossible de se décharger sur les autres des conséquences de sa propre action, et il accepte donc d'en être comptable. 》

Hans JONAS ${ }^{57}$ (1903 - 1993) va à sa manière plus loin encore: " je suis responsable envers autrui non seulement en assumant mes actes à l'égard du passé mais aussi à l'égard d'un futur lointain où je ne serai même plus présent pour répondre personnellement de ces actes. "On est responsable aussi devant les générations futures. Son argument de fond, qui part de la peur des conséquences technologiques, s'appuie positivement sur le devoir être de l'homme...

\footnotetext{
54 MORIN Edgar: «Le problème d'une démocratie cognitive » éditorial de l'InterLettre Chemin Faisant - MCX-APC $n^{\circ}$ 42 Mai 2008.

${ }^{55}$ Encyclopédie Hachette MultiMedia 1998 (adapté de...)

${ }^{56}$ FEILLET Bruno, citant RAYNAUD François, extrait de « WEBER Dictionnaire d'éthique », p. 1613-1614 sur le site « Les nouvelles philosophies morales et le pluralisme éthique », http://www.discernement.com/GrandsThemes/PhilosophieMorale/NouvPhiMo.htm

${ }^{57}$ FEILLET Bruno évoquant JONAS dans « Les nouvelles philosophies morale et le pluralisme éthique », sur le site http://www.discernement.com/GrandsThemes/PhilosophieMorale/NouvPhiMo.htm
} 
L'humanité est capable de modifier radicalement ou même de détruire son environnement. De ce pouvoir résulte, un nouveau devoir de sollicitude envers la nature.

De WEBER à JONAS, on pourrait dire que dans l'éth1ique de conviction s'est opéré un glissement entre répondre de soi et de ses actes à répondre d'autrui et lui garantir la vie. Le Développement Durable trouve ses racines dans l'éthique de Hans JONAS.

\section{2/UNE ETHIQUE DE LA DISCUSSION OU COMMUNICATIONNELLE - Jürgen HABERNAS (1929)}

Après guerre, il a plaidé pour une plus grande participation des citoyens dans le débat sur la réorganisation de la société. Son concept d'un agir communicationnel permet de construire des consensus dans un dialogue de sujet à sujet, exempt de manipulation : la relation entre les acteurs se constitue dans le langage, elle ne lui préexiste pas.

Cet intérêt d'HABERMAS pour la philosophie du langage est mis en rapport avec le travail de KANT sur l'universalisation de la maxime pour en vérifier la validité. Mais il le fait autrement : " Au lieu d'imposer à tous les autres une maxime dont je veux qu'elle soit une loi universelle, je dois soumettre ma maxime à tous les autres afin d'examiner par la discussion sa prétention à l'universalité. Ainsi s'opère un glissement : le centre de gravité ne réside plus dans ce que chacun souhaite faire valoir, sans être contredit, comme étant une loi universelle, mais dans ce que tous peuvent unanimement reconnaître comme une norme universelle ${ }^{58}$

Le glissement que fait HABERMAS porte sur le fait qu'il ne suffit pas que je me dise à moi-même que la norme que j'érige en loi universelle l'est, il faut encore que d'autres le disent avec moi. Nous avons là l'apparition d'une procédure qui passe par le langage et la discussion et donc par la multirationalité. Et déjà, il nous engage aussi à réfléchir sur la diversité des cultures

\section{Quelles intentions et visions du futur selon Edgar MORIN - Vision que je partage.}

\section{$\rightarrow$ Vers une AXIOLOGIE \\ Socle des valeurs fondamentales JUSTICE, LIBERTE, EGALITE, SOLIDARITE La structure de base (Basic Structure) de la société, décrite notamment dans l'ouvrage majeur de John RAWLS (Justice et démocratie), doit permettre d'orchestrer la cohésion sociale.}

Référons nous aussi à la notion d' Humanisme critique de TZVETAN TODORV (CNRS) Il préconise l'autonomie de la personne et de la communauté, mais liée au maintien de la dimension sociale et des valeurs qui dépassent l'individu. Il s'agit de maintenir la

\footnotetext{
${ }^{58}$ HABERMAS Jürgen, Morale et communication. Conscience morale et activité communicationnelle, Cerf, Paris, 1996, p. 88. 
référence universelle sans ignorer l'importance des cultures ou des sociétés particulières. Pour lui l'exigence d'autonomie n'entraîne pas la destruction du tissu social (\# individualisme) ni le renoncement à toute valeur transcendante ( \# materialisme)

$\rightarrow$ Vers une éthique de Reliance, EDGAR MORIN

- Comment combiner l'appel à l'universel et le respect des particularités?

Ainsi donc, les configurations réticulaires de délibération, du paysage démocratique, appellent une nouvelle multi-logique, un nouveau savoir-faire ensemble et suppose une éthique de Reliance.

"La pensée complexe est la pensée qui relie. L'éthique complexe est l'éthique de Reliance. [...] Il faut, pour tous et pour chacun, pour la survie de l'humanité, reconnaître la nécessité de relier, de se relier aux nôtres, de se relier aux autres. La pensée de la complexité ouvre la voie à une pensée authentique, affranchie du dogme de la vérité absolue, elle y gagne en ouverture, elle y perd en assurance. En reliant les connaissances, elle oriente vers la Reliance entre humains, Son principe de non séparation oriente vers la solidarité. »

« Cette notion de Reliance, j'en avais besoin : cela me parait de plus en plus évident...», dit Edgar MORIN s'entretenant en 1995 avec l'inventeur du concept de Reliance, le sociologue Marcel BOLLE de BAL. ${ }^{59}$

\section{Ethique de reliance}

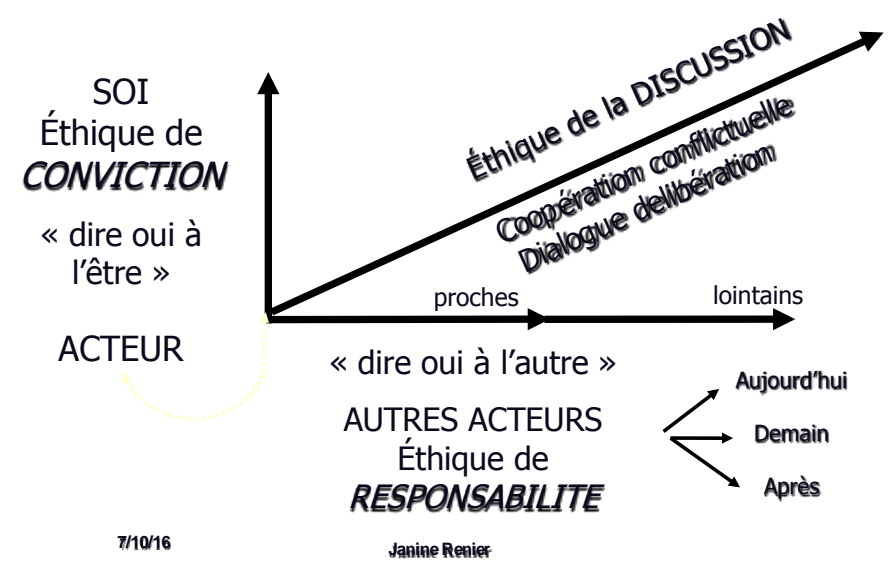

« J'aime à définir la Reliance, dans la dimension normative que je lui attribue, comme le partage des solitudes acceptées et un échange des différences respectées ${ }^{37 \text { " }}$

"Relié est passif, reliant est participant, Reliance est activant, ce concept exprimant de façon presque musicale la cellule souche de la pensée complexe. »

\footnotetext{
${ }^{59}$ BOLLE De BAL Marcel, ULB, «Reliance, Déliance, Liance, émergence de trois notions sociologiques », Sociétés $n^{\circ} 80$ -
} $2003,2$. 
Cette éthique de reliance est proche l'éthique de la collaboration préconisée par l'écologie des organisations qui prélude à la création d'un avenir commun et qui privilégie la collaboration plutôt que la concurrence

$\rightarrow$ Auto-éthique, socio-éthique, anthropo-éthique

\section{Différents niveaux de l'éthique}

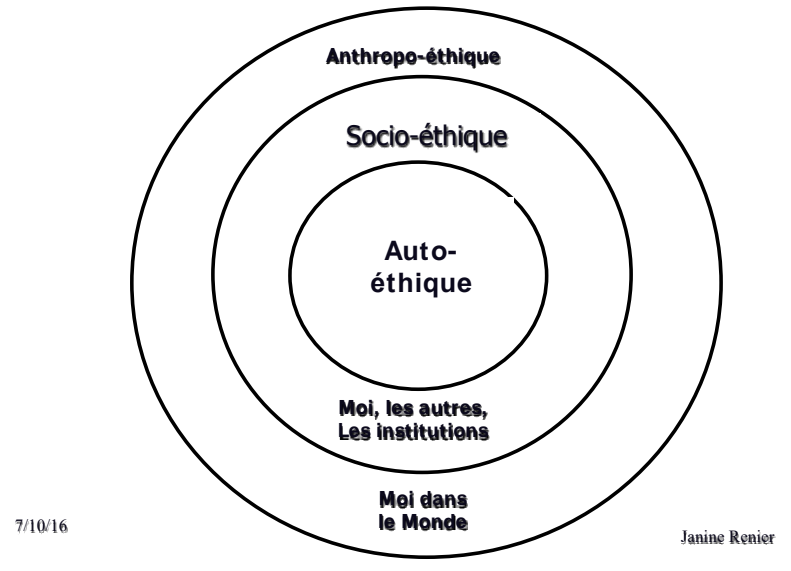

Cette co-construction ne pourrait s'opérer sans une refondation mythique ${ }^{60 \& 61}$ avec l'émergence du sens (à l'échelle planétaire) dans le cadre d'une véritable éthique de la discussion ${ }^{62}$ et de reliance ${ }^{63}$.

Dans un environnement complexe et dynamique, les théories de la contingence appellent aussi une démocratie délibérative ascendante, conjuguée à la démocratie représentative (multi niveaux, du local au mondial).

"Il s'agit bien d'accomplir l'humanité comme communauté planétaire, de prendre conscience de notre Terre patrie, de permettre à cette conscience de réaliser la citoyenneté terrienne, d'accomplir la mondialisation droits / institutions (Droits de l'homme, droits des peuples à disposer d'eux-mêmes, droit social, droit pénal mondial, etc..)»

Comment maintenant instaurer une démocratie cosmopolite, internationale, à l'heure de la mondialisation et où plongés dans une planète en danger, à ce jour, nous ne savons pas comment mettre en place cette gouvernance mondiale. Or l'urgence est criante, à côté des G8, G20, la COP 21, la COP 22 et autres mouvements alternatifs, relevant de la bonne volonté, rien n'empêche les égoïsmes nationaux de s'affronter.

D'autant plus que nous avons déjà pointé une sorte de dé-légitimation du politique face au marché, à la finance, à la bureaucratie, à la technocratie (Banque centrale européenne, Commission européenne, Banque mondiale et FMI) .Certains auteurs évoquent même à ce propos « le Sénat virtuel » qui subordonne les gouvernements à la «pensée unique» néo-libérale et par conséquent «leurs choix politiques». Mais

60 RENIER Janine citant MORIN Edgar dans «Les Mythes institutionnels » formation IEFC, Jocaste, 2015, HERSTAL. 61 HACHET Pascal: Le mensonge indispensable, du trauma social au mythe, éditions Armand Colin, Paris, 1999.

62 HABERMAS Jürgen: Morale et communication. Conscience morale et activité communicationnelle, Cerf, Paris, 1996, p. 88.

63 LE MOIGNE Jean-Louis: «Edgar Morin, le génie de la Reliance », article Synergies Monde n 4 - 2008 pp. 177-184. 
n'ignorons pas non plus les médias commerciaux, médias sociaux, juges, agences de notation, etc.

Un nouvel ordre mondial, ne devrait donc pas déboucher ni sur une « soft barbarie» des multinationales, ni sur une forme de «soft Tyrannie supranationale», prenant des décisions bureaucratiques ou technocratiques oppressantes aux niveau des politiques mais aussi des citoyens du monde, avides de plus de démocratie directe.

Pour éviter le déchirement de l'Etat Nation entre des forces contradictoires, il faudrait envisager une approche novatrice du principe de subsidiarité qui permettrait d'imaginer des réglementations supranationales pas trop envahissantes (avec une adhésion au principe universaliste), conjuguant à la fois « nouvel ordre mondial » et «autonomies locales». Comme le souligne Jean-Pierre Fitoussi : «La démocratie de marché ne désigne pas un système unique, mais plutôt un régime où le système économique obéit à une détermination politique. La variété des choix sociaux en chaque pays garantit donc la pluralité des formes que prend la démocratie de marché. "64

\section{4) Renouveau démocratique 65 : \\ comment penser - agir la démocratie et la citoyenneté dans la complexité ? Quelles organis'actions ${ }^{66}$ ?}

Comment aller vers un renouveau démocratique? Plusieurs voies sont possibles et se dessinent actuellement, Loï BLONDIAUX propose plusieurs voies de réformes... que sont en gestation, éclatées souvent... que nous devrons approfondir. ...lors de ces journées de réflexion.

- La voie de la réforme institutionnelle, la plus radicale

- Remettre en cause le processus de professionnalisation politique, Lutter contre le cumul des mandats, instaurer des règles éthiques et de contrôle plus strictes.

- Usage du tirage au sort pour certains organes, Didier VAN REYBROUCK ${ }^{67}$ nous parle des expériences positives menées en Colombie Britannique, aux Pays Bas, en Ontario, en Islande (élaboration des grandes lignes d'une constitution), en Irlande.

\footnotetext{
${ }^{64}$ FITOUSSI J.P., La démocratie et le marché, Grasset, 2004, p. 74

${ }^{65}$ BLONDIAUX Loïc, «Métamorphoses de la démocratie: vers une démocratie réelle» Session 2011 des Semaines sociales, "la démocratie une idée neuve». Professeur des Universités au département de science politique de la Sorbonne (Paris I), chercheur au Centre européen de sociologie et de science politique de la Sorbonne (CESSP) et au Centre de recherches politiques de la Sorbonne (CRPS).

${ }^{66}$ MUKUNGU KAKANGU MARIUS: GLOSSAIRE, CNRS EDITIONS HERMÈS, LA REVUE, 2011/2 № 60 , 232 À 237. «Organisaction (organisation active) : Ce néologisme est la concaténation des mots « organisation active», formé par Edgar Morin pour spécifier un type particulier d'organisation. Comme l'indique son suffixe (action), l'organis-action fait appel àl'action »

${ }^{67}$ VAN REYBROUCK David, Contre les élections, ACTES SUD/ De Bezige Bij, pour la traduction française, 2014.
} 
- Usage pour certains du référendum

- La voie de la contre - démocratie ou démocratie « sauvage »

1) Rôle d'interpellation, de critique et de vigilance des citoyens, le sommeil de la citoyenneté pouvant générer des « monstres»

Nombreux collectifs locaux mais aussi de médias sociaux à l'échelle planétaire en réseaux, pétitions: altermondialistes, Travail social militant... etc ...

2) Innovations auto-organisatrices innovantes; Occupiers, Indignatos, les Indignés, Nuit Debout, qui restent rebelles à l'institutionnalisation

3) Mécanismes d'Empowerment au niveau des enclaves locales, mais difficilement transposables à l'échelon supérieur

- La voie de la démocratie délibérative

1) Avec comme adjuvant la démocratie cognitive ${ }^{68}$ et l'organis'action

\section{- Démocratie cognitive :}

Or le problème crucial de notre temps est celui de la nécessité d'une pensée apte à relever le défi de la complexité du réel, c'est-à-dire de saisir les liaisons, interactions et implications mutuelles, les phénomènes multidimensionnels, les réalités qui sont à la fois solidaires et conflictuelles (comme la démocratie elle-même, qui est le système qui se nourrit d'antagonismes tout en les régulant).

Une connaissance où serait donc présente une pensée complexe ... (à l'école, dans les collectifs et UE d'été citoyennes... Etc) que nous n'avons pas cessé de développer dans cet article

\section{- Démocratie délibérative}

La démocratie délibérative part du principe que l'élection ne suffit plus pour produire de la légitimité. Débat préalable et inclusif à la décision.

Le fait d'autorité, le discours d'autorité ne fonctionnent plus, les citoyens veulent être entendus et associés à la discussion.

La délibération permet d'organiser la controverse, d'organiser le conflit. La démocratie délibérative répond à trois principes:

1/un principe d'inclusion, tous ceux qui sont affectés par une décision doivent pouvoir participer à cette délibération:

2/un principe d'argumentation, il faut faire en sorte que l'échange d'arguments se déploie:

$3 /$ un principe de publicité, tout cela doit se faire en public, et non plus dans un huis-clos entre experts, groupes d'intérêt et élus.

Dans le nouvel esprit de la démocratie: exemples: jurys de citoyens - tirage des citoyens au sort, on leur pose une question, ils discutent, ils auditionnent des experts et ils produisent un avis consultatif: Commission nationale du débat public, budget

${ }^{68}$ MORIN Edgard «Le problème d'une démocratie cognitive » extrait de La Méthode, Tome 6, ETHIQUE', page 171 120 
participatif de Porto Alegre au Brésil, l'assemblée citoyenne de Colombie britannique au Canada, question du nucléaire

\section{- Démocratie « Continue » dans une « Société démocratique » 69}

Le métier de citoyen ne s'arrête pas et ne se finit pas au seul moment du vote mais se poursuit en dehors des moments électoraux. "Continue " pour dire que les règles de la vie commune n'ont pas de garantie transcendantale mais restent toujours entre les mains des hommes qui, par la délibération publique, peuvent en modifier le contenu oulet le sens.

La société serait divisée en un espace civil et un espace politique.

Il est possible, dans la logique des travaux d'Habermas, de proposer un autre schéma où s'intercale, entre l'espace civil et l'espace politique, l'espace public. Lieu où se construit une opinion publique et une volonté générale sur des propositions normatives qui sont ensuite portées dans l'espace politique (propositions de règles de droit)

Cette volonté générale se forme par la délibération, par la communication des idées, par la confrontation des opinions, par l'échange d'arguments. Donc, par la discussion et de la coopération conflictuelle, loin de tout angélisme

Donc, par l'exercice et le respect des droits fondamentaux qui sont les conditions de possibilité de la discussion.

La discussion ne devient un principe actif et distinctif de la démocratie continue que par le médium du droit et, en particulier, des droits fondamentaux qui définissent le code de réalisation de l'activité communicationnelle.

\section{5) Les Plans de Cohésion Sociale - PCS en Wallonie et en Europe. Les territoires de coresponsabilités ... Together 70}

La démocratie délibérativel collaborative, un outil pour renforcer la cohésion sociale en Europe ${ }^{71}$

Pour illustrer cette capacité de démocratie continue ${ }^{72}$ ou processuelle, les projets d'agir démocratique ${ }^{73}$ modélisés par le Conseil de l'Europe (Plans de Cohésion Sociale Europe - Wallonie) sont convoqués.

\footnotetext{
${ }^{69}$ ROUSSEAU Dominique: Radicaliser la démocratie, propositions pour une refondation, Seuil, Paris, avril 2015.

70 Together https://wikispiral.org/tiki-index.php?page=New\%2OHomepage\%20EN

${ }^{71}$ ALBERT Frédéric, «La démocratie délibérative, outil pour renforcer la cohésion sociale en Europe , Mémoire de Master en gouvernance européenne, Université du Luxembourg, 26 juin 2013. P.4

72 ROUSSEAU Dominique: Radicaliser la démocratie, propositions pour une refondation, Seuil, Paris, avril 2015.

73 CONSEIL DE L'EUROPE, Construire le progrès sociétal pour le bien-être de tous avec les citoyens et les communautés, Guide méthodologique: 
Le progrès sociétal vers le bien-être de tous et la charte des responsabilités sociales partagées ${ }^{74}$

Dans sa stratégie de cohésion sociale, le Conseil de l'Europe définit cette dernière comme étant la capacité de la société à assurer le bien-être de tous par la coresponsabilité de ses différentes parties prenantes (acteurs publics, privés, citoyens, entreprises). Un lien est établi avec le développement durable en y incluant le bien-être des générations futures, faisant du bien-être de toutes les générations un objectif de progrès sociétal.

Ils mettent ensemble en place des actions pilotes de coresponsabilités La mise en place de cette stratégie suppose l'emploi de la méthodologie SPIRAL à la fois au niveau local, régional et européen en développant la notion de territoires de coresponsabilité

L'objectif de SPIRAL ${ }^{75}$ est de permettre, grâce à la contribution de tous, de surpasser progressivement les difficultés pour que la construction de la coresponsabilité pour le bien-être de tous devienne un chemin plus naturel dans nos sociétés et soit reconnue comme un élément clé de notre avenir commun. Et se base sur les principes de coconstruction

La méthodologie SPIRAL, entièrement ouverte et systématisée, est basée sur des principes de démocratie directe et de démocratie collaborative. Complémentaires de la démocratie représentative elles apportent des réponses essentielles aux limites de cette dernière, notamment en créant les conditions d'une expression directe des citoyens et d'un dialogue transversal qui font souvent défaut dans nos sociétés

\section{L'agir démocratique peut s'exprimer de la manière suivante}

- La capacité à réfléchir ensemble,

- La capacité de définir les responsabilités de chacun,

- La capacité de co-décider.

- La capacité de tirer et capitaliser les enseignements

\footnotetext{
${ }^{74}$ Recommandation CM/Rec(2014)1 du Comité des Ministres aux Etats membres relative à la Charte du Conseil de l'Europe sur les responsabilités sociales partagées https://search.coe.int/cm/Pages/result_details.aspx?ObjectID=09000016805c6827
} 


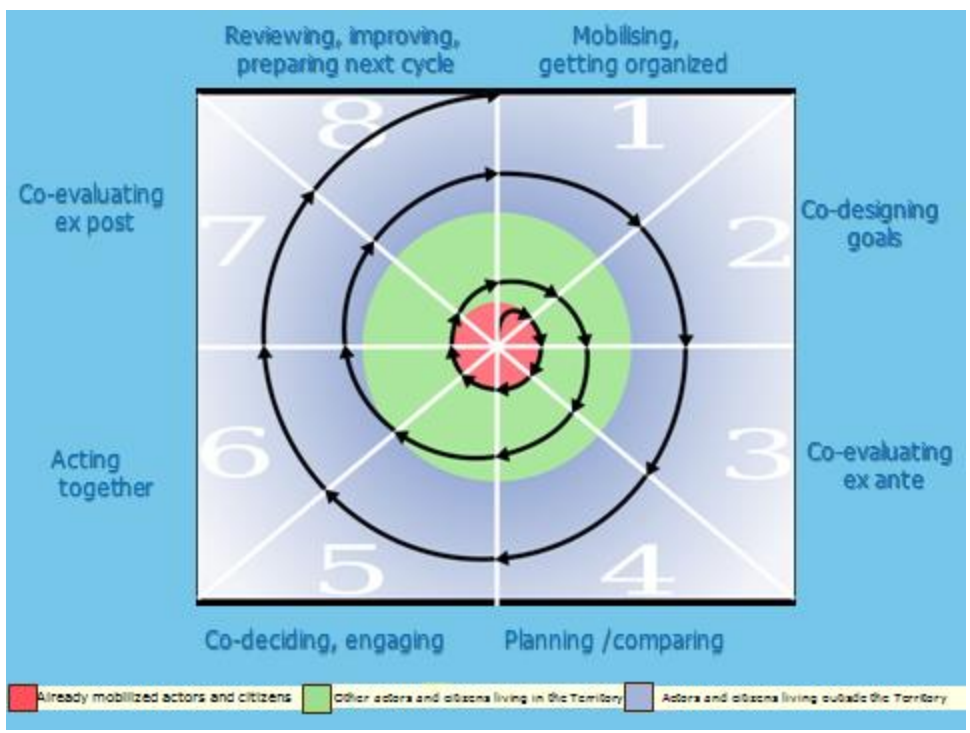

La région wallonne de Belgique ${ }^{76}$, impliquée dans ce projet depuis plus de dix ans, Cette dernière, a participé depuis les années 2000, à la « lutte pour une meilleure intégration sociale et le renforcement de la cohésion sociale », en utilisant la méthodologie du CoE.

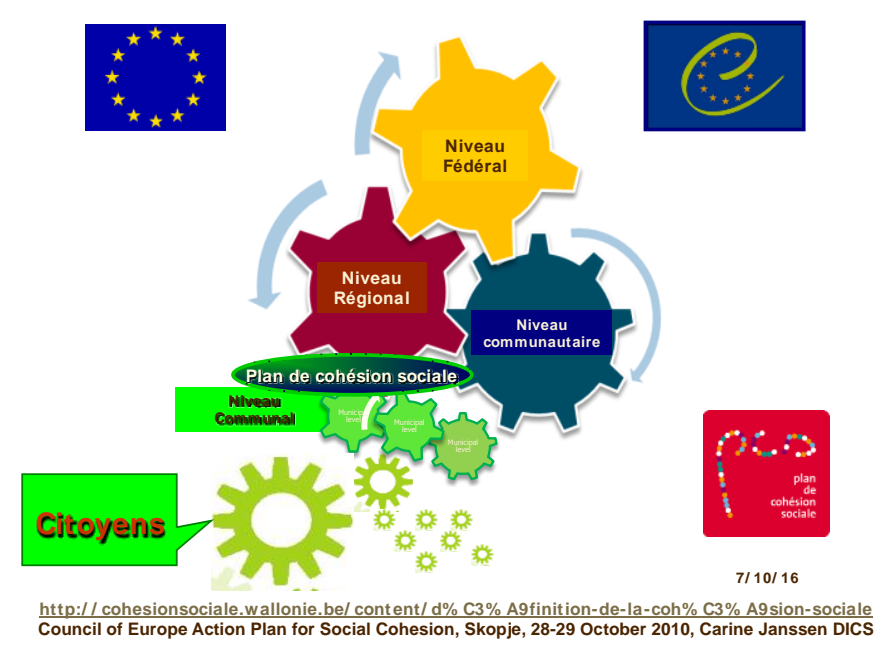

Le cheminement, se réalise à travers la défense et la recherche des droits fondamentaux, tels que protégés par la constitution belge et la Convention Européenne des Droits de l'Homme (CEDH).

Les 1700 actions pilotes comptabilisées à l'heure actuelle, s'inspirent toutes de la définition de la cohésion sociale du CoE, qui vise la « recherche du bien-être pour tous dans la durée».

Mais elles s'inspirent aussi de différentes approches méthodologiques participatives qui

76 JANSSEN Carine, DICS, http://cohesionsociale.wallonie.be/content/définition-de-la-cohésion-sociale 
émergent du terrain, notamment celle proposée par «le Village systémique »i77: l'approche Reflect Action, qui s'ancre dans des pratiques de conscientisation populaire inspirées de Paolo Freire.

Il s'agit bien de vivre une démarche de co-construction du bien-être individuel et collectif pour initier un changement sociétal «Partant d'activités créatives et participatives, nous allons observer nos fonctionnements et ressentis. Nous les analyserons pour pouvoir agir concrètement sur le fonctionnement de la société et aller en coresponsabilité vers le bien-être de tous aujourd'hui et demain. "

\section{6) Quel Devenir ? \\ Quelle Démocratie? DEMAIN ?}

\section{Le défi de ces méta-morphoses est donc inséparablement éthique, épistémologique et organis'actionnel !}

Et ce défi ne pourra être relevé qu'en co-construction dans une société globalement démocratique.

Penser le progrès en termes de complexité et de capacité à vivre ensemble sur une même planète est devenu, plus que jamais dans l'histoire de l'humanité, à la fois une évidence et une nécessité, mais saurons réagir nous à temps et construire un «DEMAIN» qui nous ouvrira à «d'autres possibles» vers une authentique démocratie?

Edouard DELRUELLE ${ }^{78}$ décrit le projet de philosophie politique de Cornélius CASTORIADIS : "celui d'une autonomie radicale de l'individu et de la société, ce qui suppose d'assumer le paradoxe que le sujet est « institué » par la société, et qu'il ne se maintient dans l'autonomie que par sa participation active et critique à cette institution sociale. Cette autonomie est donc tout l'inverse de l'individu narcissique et jouissif créé par la société capitaliste, dont il restera jusqu'à la fin le pourfendeur sans pitié. ". Face aux responsables politiques de plus en plus disqualifiés et dépossédés d'une partie de leur pouvoir à l'aune de la Finance et du Marché, il est urgent d' inventer ensemble une nouvelle alliance: politiques re-légitimés, citoyens dont les usagers, gestionnaires, travailleurs dont les assistants sociaux, chercheurs, experts, étudiants et témoins, dans une sorte de boucle récursive démocratique auto-éco-instituante de nos sociétés.

\footnotetext{
${ }^{77}$ Le Village Systémique, http://new.systemique.levillage.org

${ }^{78}$ DELRUELLE E., De l'homme et du citoyen, Une introduction à la philosophie politique, »éditions de Boeck, Louvain-LaNeuve,2014, pp 252. 


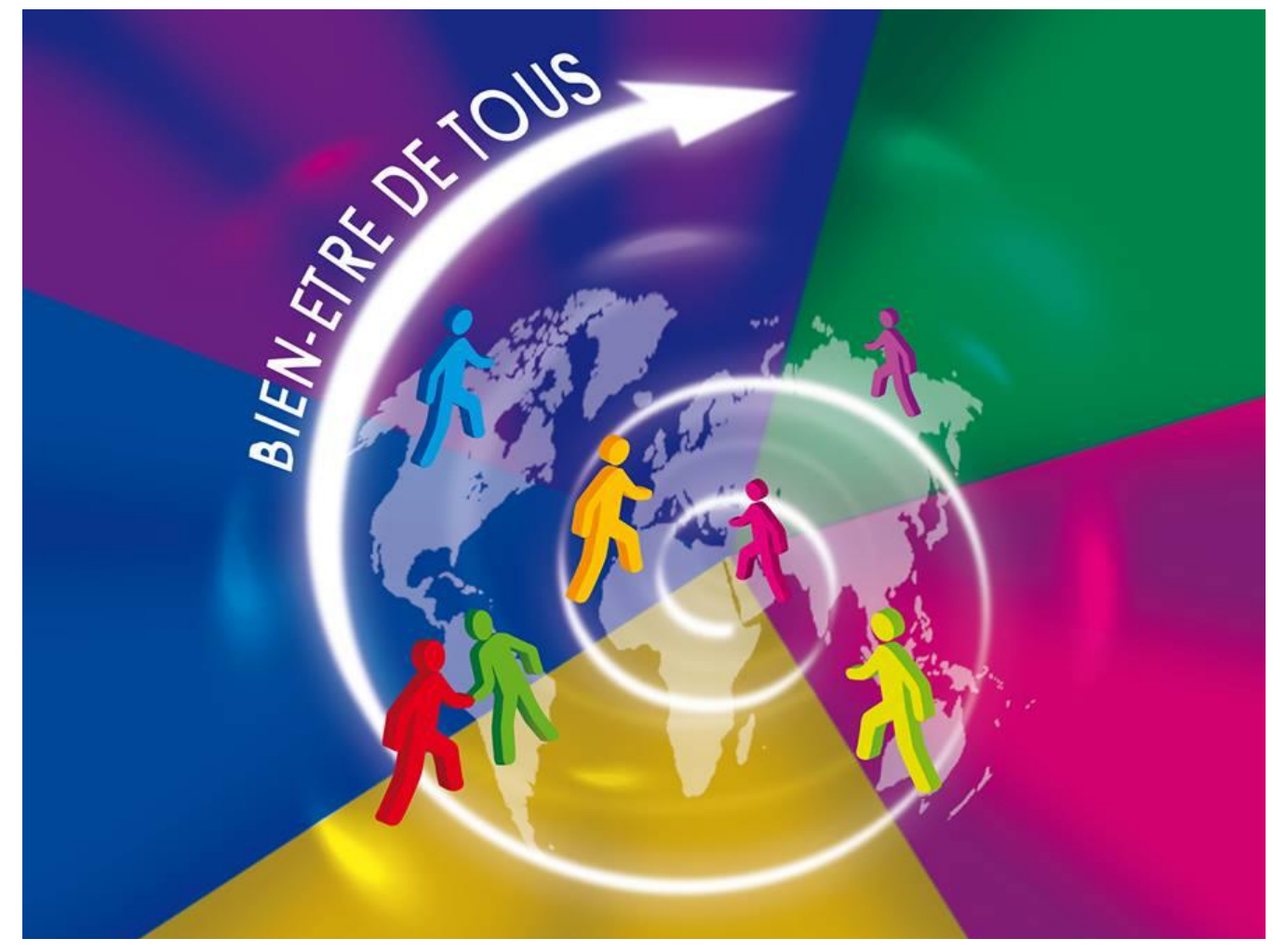

